Division of Geological \& Geophysical Surveys

RAW-DATA FILE 2002-3

\title{
MAJOR OXIDE, MONOR OXIDE, TRACE ELEMENT, AND GEOCHEMUCAL DATA FROM ROCKS COLLECTED IN THE BIG DELTA QUADRANGLE, ALASKA IN 2001
}

by

J.E. Athey, M.B. Werdon, D.J. Szumigala, R.J. Newberry, and M.R. Johnson

$\$ 15.00$

June 2002

THIS REPORT HAS NOT BEEN REVIEWED FOR TECHNICAL CONTENT OR FOR CONFORMITY TO THE

EDITORIAL STANDARDS OF DGGS

Released by

STATE OF ALASKA

DEPARTMENT OF NATURAL RESOURCES

Division of Geological \& Geophysical Surveys

794 University Avenue, Suite 200

Fairbanks, Alaska 99709-3645 


\section{CONTENTS}

PAGE

INTRODUCTION

ANALYTICAL METHODS

\section{TABLES}

TABLE 1. CONCENTRATION OF MAJOR OXIDES, MINOR OXIDES, AND TRACE ELEMENTS IN ROCK SAMPLES COLLECTED IN THE BIG DELTA QUADRANGLE.

TABLE 2. LOCATION AND DESCRIPTION OF ROCK SAMPLES COLLECTED FOR MAJOR OXIDE, MINOR OXIDE, AND TRACE ELEMENT ANALYSES IN THE BIG DELTA QUADRANGLE

TABLE 3. CONCENTRATION OF TRACE ELEMENTS IN ROCK SAMPLES COLLECTED IN THE BIG DELTA QUADRANGLE

TABLE 4. LOCATION AND DESCRIPTION OF ROCK SAMPLES COLLECTED FOR TRACE ELEMENT ANALYSES IN THE BIG DELTA QUADRANGLE.

TABLE 5. LIMITS AND ANALYTICAL METHODS FOR MAJOR-OXIDE, MINOR-OXIDE, AND TRACE ELEMENT ANALYSES

TABLE 6. LIMITS AND ANALYTICAL METHODS FOR TRACE-ELEMENT GEOCHEMICAL ANALYSES

\section{SHEETS}

SHEET 1. LOCATION MAP OF ROCK SAMPLES ANALYZED FOR MAJOR-OXIDES, BIG DELTA B-2, B-3, B-4, C-2, C-3, C-4 QUADRANGLES, ALASKA IN 2001, I SHEET (IN POCKET)

SHEET 2. LOCATION MAP OF ROCK SAMPLES ANALYZED FOR TRACE ELEMENT GEOCHEMISTRY, BIG DELTA B-2, B-3, B-4, C-2, C-3, C-4 QUADRANGLES, ALASKA IN 2001, I SHEET (IN POCKET)

Note: This report (including all analytical data, tables, and map sheets) is available in digital format from the DGGS web site (http://wwwdggs.dnr.state.ak.us) at no charge. The digital data are available as PDF files and Excel spreadstheets. 


\title{
MAJOR OXIDE, MINOR OXIDE, TRACE ELEMENT, AND GEOCHEMICAL DATA FROM ROCKS COLLECTED IN THE BIG DELTA QUADRANGLE, ALASKA IN 2001
}

\author{
by \\ J.E. Athey, M.B. Werdon, D.J. Szumigala, And R.J. Newberry, and M.R. Johnson
}

INTRODUCTION

Mineral resource personnel from the Alaska Division of Geological \& Geophysical Surveys carried out a geological field survey, including mapping and sampling in the Big Delta quadrangle, Alaska from August 7-2I, 200I. The fieldwork provides basic information critical to building an understanding of Alaska's geology and is part of an integrated program of airborne geophysical surveys followed by geological mapping programs. During 2001, 68 rock samples were collected for geochemical trace-element analysis, and 122 samples were collected for whole rock (major and minor oxides, and petrogenetically important trace element data) analysis. The locations of these samples are shown on Sheets 1 (whole rack) and 2 (trace-element geochemistry). Location data (in UTM coordinates based on Clark 1866 spheroid. NAD27 datum, UTM zone 6 projection), descriptions, and analytical results for each sample are tabulated in tables $1,2,3$, and 4 .

\section{ANALYTICAL METHODS}

All whole rock analyses (table 1) were performed by Chemex Labs, Inc. Major and minor element oxides were determined by X-ray fluorescence spectrometry (XRF) following a lithium tetraborate fusion. Trace elements (Ba, $\mathrm{Nb}, \mathrm{Rb}, \mathrm{Sr}, \mathrm{Y}$, and $\mathrm{Zr}$ ) were also analyzed using $\mathrm{XRF}$ methods on a pressed powder pellet. Analytical detection limits are tabulated in table 5 .

All 2001 trace-element geochemical analyses (table 3) were performed by Chemex Labs, Inc. Rock samples were crushed so that at least 70 percent of the material passed through a -10 (2 mm) mesh screen. Representative aliquots of 200 grams and 30 grams each were taken using a stainless steel riffle splitter. These samples were then pulverized in a chrome steel ring mill so that 85 percent of the sample passed through a -200 ( 75 micron) mesh screen. Most trace element analyses were performed on the 200 gram split while gold, platinum and palladium analyses were performed on the 30 gram representative split.

Most samples were analyzed for gold using atomic absorption spectroscopy following fire assay fusion (FA-AAS). A few gold analyses and all platinum and palladium analyses were done by inductively coupled plasma - mass spectrometry (ICP-MS) following a fire assay fusion. All other trace element geochemical analyses were performed by inductively coupled plasma - atomic emission spectroscopy (ICP-AES) methods after nitric aqua regia digestion. This method of digestion is possibly incomplete for some elements and may result in lower analytical results for certain elements. The elements that may be affected by incomplete digestion as well as analytical methods, detection limits and upper limits are tabulated in table 6. 
Table 1. Concentration of major-oxides, minor oxides, and trace elements in rock samples collected in the Big Delta Quadrangle. note: -- $=$ not analyzed

\begin{tabular}{|c|c|c|c|c|c|c|c|c|c|c|c|c|c|c|c|c|c|c|c|c|c|c|}
\hline SAMPLE & $\begin{array}{c}A / 203 \\
\%\end{array}$ & $\begin{array}{c}\mathrm{BaO} \\
\%\end{array}$ & $\begin{array}{c}\mathrm{CaO} \\
\%\end{array}$ & $\begin{array}{c}\mathrm{Cr} 2 \mathrm{O}_{3} \\
\%\end{array}$ & $\begin{array}{c}\text { FeO } \\
\%\end{array}$ & $\begin{array}{c}\mathrm{Fe} 2 \mathrm{O} 3 \\
\%\end{array}$ & $\begin{array}{c}\mathrm{K} 2 \mathrm{O} \\
\%\end{array}$ & $\begin{array}{c}\mathrm{MgO} \\
\%\end{array}$ & $\begin{array}{c}\text { Mno } \\
\%\end{array}$ & $\underset{\%}{\mathrm{Na} 2 \mathrm{O}}$ & $\begin{array}{c}\mathrm{P} 2 \mathrm{O} 5 \\
\%\end{array}$ & $\begin{array}{c}\mathrm{SiO} 2 \\
\%\end{array}$ & $\begin{array}{l}\text { Sro } \\
\%\end{array}$ & $\begin{array}{c}\mathrm{TiO} 2 \\
\%\end{array}$ & $\begin{array}{l}\text { LOI } \\
\%\end{array}$ & TOTAL & $\begin{array}{l}\mathrm{Ba} \\
\mathrm{ppon}\end{array}$ & $\begin{array}{l}\mathrm{Rb} \\
\mathrm{ppm}\end{array}$ & $\begin{array}{c}\mathrm{Sr} \\
\mathrm{ppm}\end{array}$ & $\begin{array}{l}\mathrm{Nb} \\
\mathrm{ppm}\end{array}$ & $\begin{array}{c}\mathrm{Zr} \\
\mathrm{ppm}\end{array}$ & $\begin{array}{c}\mathrm{Y} \\
\mathrm{ppm}\end{array}$ \\
\hline 01.JEA375A & 14.09 & 0.01 & 0.98 & $<.01$ & $\ldots$ & 1.33 & 4,48 & 0.22 & 0.04 & 2.54 & 0.2 & 73.7 & 0.01 & 0.15 & 0.81 & 98.56 & 340 & 184 & 80 & 14 & 63 & 24 \\
\hline 01JEA381A & 1.82 & 02 & 1.14 & 01 & - & 29 & 3.44 & 0.86 & 0.03 & 2.74 & $0 . \overline{11}$ & 72.51 & 0.02 & 0.23 & 1.13 & 99.45 & 380 & 136 & 140 & 20 & 78 & 28 \\
\hline 01JEA400A & 68 & 06 & 3.02 & & - & 3.64 & 2.94 & t.1 & 0.06 & 2.53 & 0.1 & 69.17 & 0.03 & 0.47 & 0.89 & 99.7 & 730 & 120 & 196 & 16 & 150 & 30 \\
\hline $\mathrm{A} 403 \mathrm{~A}$ & 22.01 & 14 & 0.74 & & - & 7.56 & 4.89 & 1.97 & 0.07 & 1.6 & 0.16 & 54.98 & 0.01 & 0.8 & 4.38 & 99.32 & 1310 & 176 & 166 & 28 & 144 & 40 \\
\hline $405 \mathrm{~A}$ & 4.46 & 04 & 8.42 & 01 & - & 12.8 & 1.63 & 4.7 & 0.21 & 3.45 & 0.51 & 49.67 & 0.03 & 2.58 & 1.54 & 9.74 & 470 & 58 & 288 & 38 & 234 & 36 \\
\hline $412 \mathrm{~A}$ & 12.82 & 05 & 0.89 & $<.01$ & - & 2.06 & 4.21 & 0.19 & 0.02 & 2.11 & 0.08 & 76.22 & $<.01$ & 0.31 & 0.68 & 99.64 & 650 & 200 & 50 & 12 & 165 & 40 \\
\hline & 14.9 & 01 & 9.72 & & - & 1066 & 1,23 & 6.09 & 0.15 & 3.33 & 22 & 50.79 & 0.02 & 1.72 & 0.93 & 77 & 165 & 32 & 226 & 26 & 174 & 26 \\
\hline $32 \mathrm{~A}$ & 4.34 & 06 & 1.09 & & - & 5.4 & 3.26 & 1.83 & 0.08 & 0.93 & 0.09 & 69.71 & 0.01 & 0.59 & 1.89 & 28 & 665 & 128 & 116 & 16 & 189 & 28 \\
\hline $4437 \mathrm{~A}$ & & 11 & 7.35 & $<.01$ & - & 13.77 & 37 & 3.98 & 0.21 & 3.08 & 54 & 50.99 & 0.03 & 3.68 & 2.65 & 9.5 & 170 & 26 & 298 & 34 & 276 & 40 \\
\hline 01JE & 13.17 & 02 & 0.57 & & - & 1.41 & 5.13 & 0.08 & 0.03 & 2.6 & 03 & 76.24 & 0.01 & 0.13 & $0.4 \mathrm{~B}$ & 9.9 & 385 & 162 & 108 & 30 & 111 & 30 \\
\hline $\mathrm{A} 447 \mathrm{~A}$ & 14.38 & 13 & 1.94 & .01 & - & 2.14 & 3.79 & 0.64 & 0.06 & 2.97 & 09 & 72.24 & 0.04 & 0.32 & 0.67 & .41 & 1335 & 158 & 338 & 18 & 141 & 24 \\
\hline & 14.04 & 1 & 3.22 & & - & 62 & 84 & 12 & 0.02 & 2.41 & 11 & 74.23 & 0.02 & 0.06 & 0.6 & 39 & 870 & 104 & 158 & 10 & 42 & 22 \\
\hline $88 \mathrm{~A}$ & 8.9 & 0.03 & 78 & 0.01 & - & 3.55 & 1.83 & 0.96 & 0.05 & 2.26 & 0.05 & 79.16 & 0.01 & 0,44 & 1.05 & 08 & 515 & 74 & 102 & 14. & 255 & 20 \\
\hline $8 \mathrm{~A}$ & 13.96 & .01 & 10.15 & & - & 12.37 & 0.09 & 02 & 0.19 & 2.72 & 28 & 49.14 & 0.04 & 2.24 & 2.74 & & 50 & 14 & 420 & 20 & 156 & 26 \\
\hline & & & & & - & 1.69 & 35 & & 0.01 & & & 90.94 & 6.01 & 0.19 & 0.9 & 68 & 110 & 30 & 12 & 12 & 174 & 18 \\
\hline $527 \mathrm{~A}$ & 14 & 01 & 3.01 & 0.01 & - & 4.81 & 2.07 & 1.82 & 0.08 & 1.68 & 0.07 & 70.49 & 0.01 & 0.52 & 1 & & 465 & 102 & 378 & 16 & 114 & 28 \\
\hline $35 \mathrm{~A}$ & 4.63 & 0.1 & 1.62 & & - & 2.02 & 3.64 & 0.4 & 0.04 & 2.67 & 0.1 & 73.55 & 0.03 & 0.22 & 0.75 & & 1046 & 136 & 220 & 16 & 138 & 26 \\
\hline & & 06 & & & - & 83 & 3.37 & 2.06 & 0.1 & 1.56 & 0.18 & 59.65 & 0.01 & 1.35 & 2.76 & 9.7 & $\cdots$ & - & - & $\ldots$ & - & $\cdots$ \\
\hline $643 \mathrm{~A}$ & 19 & 17 & 53 & 0 & $\ldots$ & 5.14 & 4.16 & 1.78 & 0.06 & 2.7 & 0.07 & 64.39 & 0.05 & 0.73 & 1.74 & .72 & $=$ & | & - & -- & - & - \\
\hline & & & & & - & 6.5 & & & 0.11 & & & 59.63 & 0.05 & 0.86 & 1.34 & & 1485 & 96 & 440 & 16 & $165]$ & 22 \\
\hline $01 \mathrm{JI}$ & 87 & 12 & 1.69 & & $-\cdots$ & 6.96 & 3.22 & 2.14 & 0.08 & 1.72 & 0.08 & 66.25 & 0.01 & 0.98 & 1.5 & 63 & 1180 & 142 & 186 & 28 & 300 & 30 \\
\hline $74 \mathrm{~A}$ & & & & & $-\infty$ & 1.1 & & & 0.01 & 2.47 & 0.14 & 74 & $<01$ & 0.1 & 0.99 & & 310 & 82 & 66 & 10 & 60 & 28 \\
\hline $01 \mathrm{ME}$ & 14.01 & 0.11 & 1.98 & & $=$ & 2.48 & 3.42 & 0.56 & 0.04 & 2.71 & $0 . \overline{13}$ & 71.93 & 0.04 & 0.3 & 0.83 & 55 & 1030 & 112 & 280 & 18 & 159 & 24 \\
\hline 01MBW486A & 16.03 & 0.04 & 3.79 & 0.01 & - & 4.26 & 2.51 & 1.43 & 0.08 & 3.37 & 0.13 & 65.98 & 0.03 & 0.57 & 0.7 & & 600 & 124 & 244 & 18 & 165 & 30 \\
\hline $8 \mathrm{BA}$ & & & & & $-\infty+\infty$ & 3.25 & 5.18 & 0.99 & 0.07 & & & 72.37 & 0.01 & 0.49 & 1.3 & 71 & 880 & 134: & 164 & 20 & 252 & 34 \\
\hline 01MBW494A & 552 & 0.05 & 6.15 & $<.01$ & - & 7.68 & 2.91 & 3.3 & 0.1 & 2.46 & 0.17 & 53.38 & 0.04 & 1.05 & 2.16 & 99.97 & 640 & 156 & 346 & 16 & 144 & 28 \\
\hline DIMBW496A & 17.5 & 0.08 & 3.92 & & - & 4.14 & 3.37 & 1.67 & 0.07 & 3.66 & 0.2 & 62.3 & 0.04 & 0.66 & 0.95 & & 855 & 158 & 338 & 18 & 189 & 26 \\
\hline O1MBN512A & & 0.06 & 3.28 & & $\ldots$ & 3.74 & 3.06 & 1.27 & 0.07 & 2.95 & 0.13 & 68.02 & 0.03 & 0.46 & 1.16 & 99.86 & 700 & 114 & 274 & 16 & 141 & 24 \\
\hline D1MBW516A & 13.55 & 0.15 & 1.4 & & - & 3.05 & 5.75 & 0.58 & 0.04 & 2.21 & 0.18 & 70.84 & 0.02 & 0.42 & 0.57 & 98.76 & 1260 & 180 & 158 & 20 & 186 & 42 \\
\hline OIMBW519C & 16.84 & 0.11 & 3.06 & & - & 3.58 & 3.29 & 1.58 & 0.06 & 3.55 & 0.15 & 65.95 & 0.04 & 0.6 & 0.91 & 99.72 & 1020 & 148 & 314 & 20 & 192 & 22 \\
\hline 01MBW528A & & 0.06 & 2.14 & & -- & 3.92 & 3.62 & 1.13 & 0.06 & 2.29 & 0.1 & 69.64 & 0.01 & 0.39 & 1.47 & 98.43 & 730 & 150 & 152 & 22 & 165 & 36 \\
\hline $01 \mathrm{MBWS34A}$ & 13.64 & 0.07 & 1.4 & 0.01 & -- & 3.56 & 3.81 & 1,02 & 0.06 & 2.46 & 0.09 & 70.43 & 0.01 & 0.36 & 1.68 & 98.6 & 780 & 152 & 160 & 22 & 162 & 36 \\
\hline O1MBW541B & 15.18 & 0.09 & 2.93 & 0.01 & -1 & 3.85 & 3.35 & 2 & 0.07 & 2.9 & 0.1 & 66.53 & 0.03 & 0.5 & 2.06 & 99.6 & 865 & 154 & 256 & 16 & 141 & 28 \\
\hline
\end{tabular}


Table 1. (continued)

\begin{tabular}{|c|c|c|c|c|c|c|c|c|c|c|c|c|c|c|c|c|c|c|c|c|c|c|}
\hline SAMPLE & $\begin{array}{c}\mathrm{Al} 2 \mathrm{O} 3 \\
\% \\
\end{array}$ & $\begin{array}{c}\text { BaO } \\
\%\end{array}$ & $\begin{array}{c}\mathrm{CaO} \\
\%\end{array}$ & $\begin{array}{c}\mathrm{Cr} 2 \mathrm{O}_{3} \\
\%\end{array}$ & $\begin{array}{c}\text { Feo } \\
\%\end{array}$ & $\begin{array}{c}\mathrm{Fe} 203 \\
\%\end{array}$ & $\begin{array}{c}K 20 \\
\%\end{array}$ & $\begin{array}{c}\mathrm{MgO} \\
\% \\
\end{array}$ & $\begin{array}{c}\text { MnO } \\
\%\end{array}$ & $\begin{array}{c}\mathrm{Na} 2 \mathrm{O} \\
\%\end{array}$ & $\begin{array}{c}P 205 \\
\%\end{array}$ & $\begin{array}{c}\mathrm{SiO} 2 \\
\%\end{array}$ & $\begin{array}{c}\text { Sro } \\
\%\end{array}$ & $\begin{array}{c}\mathrm{THO}^{2} \\
\%\end{array}$ & $\begin{array}{c}\text { LO: } \\
\%\end{array}$ & TTAL & $\begin{array}{c}\text { Ba } \\
\mathrm{ppm}\end{array}$ & $\begin{array}{l}\mathrm{Rb} \\
\mathrm{pom}\end{array}$ & $\begin{array}{c}\mathrm{Sr} \\
\mathrm{ppm}\end{array}$ & $\begin{array}{l}\text { Nb } \\
\text { ppm }\end{array}$ & $\begin{array}{c}\mathrm{Zr} \\
\mathrm{ppm}\end{array}$ & $\begin{array}{c}Y \\
\mathrm{ppm}\end{array}$ \\
\hline $01 M B W 5428$ & 15.35 & 0.08 & 7.17 & 0.01 & $\ldots$ & 7.39 & 2.39 & 7.79 & 0.13 & 2.98 & 0.12 & 53.13 & 0.06 & 0.68 & 1.81 & 99.09 & 795 & 96 & 510 & 12 & 117 & 22 \\
\hline BW585B & 14.43 & 0.07 & .08 & 0.01 & - & 2.07 & 2.73 & 0.26 & 03 & 276 & 0.08 & 74.18 & $<.01$ & 0.32 & 2.48 & 99.49 & 695 & 170 & 70 & 30 & 159 & 18 \\
\hline 01 MBW587A & 13.91 & 0.01 & 10.25 & 0.01 & - & 12.79 & 0.09 & 6.14 & 0.18 & 1.84 & 0.29 & 48.18 & 0.06 & 2.27 & 3.57 & 99.59 & 185 & 14 & 592 & 18 & 159 & 28 \\
\hline $01 \mathrm{MBW} 613 \mathrm{~A}$ & 14.18 & 01 & 0.48 & $<.01$ & - & 0.96 & 3.54 & $<.01$ & 0.04 & 3.8 & $0 . \overline{22}$ & 74.79 & $<.01$ & 0.07 & 0.81 & 98.89 & 65 & 278 & $<2$ & 16 & 42 & 16 \\
\hline $666 \mathrm{~A}$ & 11.16 & 0.09 & 12.9 & 0.01 & $\ldots$ & 13.26 & 1.27 & 10.57 & 0.21 & 1.56 & 0.89 & 42.62 & 0.06 & 2.86 & 2.2 & 99.66 & 830 & 48 & 554 & 138 & 288 & 36 \\
\hline & 10.5 & 0.01 & 9.75 & 0.01 & - & 13.56 & 0.75 & 9.07 & 0.17 & 0.46 & 0.54 & 48.84 & $<.01$ & 3.86 & 2.04 & 99.56 & 180 & 42 & 72 & 60 & 276 & 30 \\
\hline $3 \mathrm{~A}$ & 5.88 & 0.06 & 1.61 & $<\overline{01}$ & - & $\{.81$ & 4.11 & 0.44 & 0.03 & 3.37 & 0.11 & 71.32 & 0.03 & 0.35 & 0.65 & 99.77 & 685 & 266 & 222 & 38 & 156 & 20 \\
\hline & & 01 & 57 & 0.01 & - & 47 & 087 & 11.72 & 0.2 & .47 & 48 & 47.64 & 0.01 & 2.62 & 1.71 & 98.93 & 270 & 30 & 88 & 46 & 192 & 22 \\
\hline $38 \mathrm{~A}$ & 11.55 & 0.04 & 98 & 0.01 & 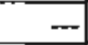 & 66 & 0.91 & 11.1 & 0.19 & 1.51 & 0.57 & 44.31 & 0.05 & 2.61 & 1.19 & 99.68 & 465 & 3 & 456 & 90 & 201 & 20 \\
\hline$\overline{01 M}$ & 6.89 & $<.01$ & 22 & 0.01 & - & 10.68 & 0.25 & $\$ 1.22$ & 0.22 & 0.95 & $0 . \overline{37}$ & 37.29 & 0.09 & 2.4 & 9.07 & 99.66 & 80 & 18 & 748 & 38 & 168 & 22 \\
\hline $716 \mathrm{~A}$ & 10.67 & 0.03 & 11.95 & 0.01 & - & 12.68 & 0.79 & 11.45 & 0.18 & 1.8 & 0.47 & 44.72 & 0.04 & \begin{tabular}{|l|l}
3.21 \\
\end{tabular} & 1.57 & 99.57 & 400 & 30 & 358 & 72 & 237 & 22 \\
\hline & 10.3 & $<.01$ & 10.79 & 0.01 & $=$ & 11.99 & 0.73 & 10.26 & 0.2 & 0.81 & 0.42 & 49.61 & 0.01 & 2.93 & 1.43 & 99.49 & $\{55$ & 34 & 112 & 54 & 219 & 22 \\
\hline & 13.25 & 0.04 & 10.1 & $<01$ & - & 12.78 & 0.83 & 6.12 & 0.26 & 2.48 & 0.17 & 46.24 & 0.02 & 6.52 & 0.82 & 99.63 & 240 & 28 & 252 & 26 & 108 & 18 \\
\hline & 1177 & 0.02 & 9.76 & 0.01 & - & 13.55 & 0.47 & 8.58 & 0.21 & 2.61 & 0.67 & 46.59 & 0.06 & 4.46 & 0.75 & 99.51 & 250 & 22 & 580 & 62 & 279 & 24 \\
\hline & 15.3 & $<.01$ & 1.46 & $\leq .01$ & - & 0.36 & 0.54 & 0.06 & $<.01$ & 4.77 & 0.01 & \begin{tabular}{|l}
76.45 \\
\end{tabular} & 0.05 & 0.05 & 0.76 & 99.81 & 35 & 22 & 402 & & 42 & 10 \\
\hline $01 \mathrm{~N}$ & 9.18 & 0.01 & $\{2.68$ & 0.01 & - & 13.76 & 0.44 & 11.37 & 0.18 & 1.42 & 0.5 & 45.17 & 0.03 & 3.28 & 1.16 & 99.19 & 110 & 18 & 294 & 50 & 225 & 22 \\
\hline 7408 & 14.41 & $<.01$ & 1.29 & $<01$ & - & 0.78 & 1.34 & 0.23 & 0.01 & 5.14 & 0.11 & 74.32 & 0.06 & 0.15 & 0.72 & 98.56 & 305 & 50 & 452 & 14 & 87 & 32 \\
\hline $\mathrm{W} / 42 \mathrm{C}$ & 15.08 & $<.01$ & 0.48 & $<.01$ & - & 0.88 & 2.11 & 0.05 & 0.16 & 3.97 & 0.05 & 74.63 & $<01$ & 0.04 & 1.14 & 98.59 & 55 & 196 & 6 & 26 & 36 & 22 \\
\hline $46 \mathrm{~A}$ & 14.43 & 0.18 & 0.72 & $<01$ & - & 4.6 & 3.87 & 2.03 & 0.08 & 1.88 & 0.11 & 69.47 & 0.02 & 0.68 & 1.54 & 99.56 & 1685 & 142 & 102 & 24 & 273 & 30 \\
\hline $53 \mathrm{~A}$ & 14.28 & $<01$ & 0.47 & $<.01$ & - & 0.57 & 3.92 & 0.04 & 0.07 & 3.77 & 0.08 & 75.98 & $<.01$ & 0.04 & 0.57 & 99.79 & 195 & 350 & 58 & 16 & 33 & 20 \\
\hline & 1449 & $<.01$ & 1.79 & 0.01 & - & 1.22 & 0.75 & 6.77 & 0.2 & 2.61 & 26 & 45.81 & 0.03 & 2.29 & 1.11 & 99.34 & 145 & 26 & 244 & 14 & 141 & 34 \\
\hline & 4.3 & 0.07 & 8.79 & 0.01 & - & 91.07 & 1.15 & 0.54 & 18 & 2.79 & 0.61 & 50.36 & 0.05 & 2.36 & 2.21 & 99.49 & 705 & 38 & 396 & 28 & 270 & 40 \\
\hline & 03 & 0.15 & & & $=$ & 4.84 & 1.59 & .41 & 0.08 & 2.77 & 0.14 & 65.85 & 0.04 & 0.66 & 1.13 & 99.57 & 1605 & 64 & 284 & 16 & 126 & 12 \\
\hline & 62 & 0.08 & 7 & 501 & - & 9.2 & 1.61 & 5.17 & 01 & 1.88 & $0 . \overline{43}$ & 51.26 & 0.04 & 2.03 & 6.24 & 99.31 & 680 & 52 & 324 & 26 & 225 & 32 \\
\hline $799 \mathrm{~A}$ & 13.77 & 0.1 & 0.97 & 0.01 & - & 3.33 & 0.5 & 1.44 & 0.04 & 5.51 & 0.09 & 72.38 & 0.01 & 0.4 & 1.25 & 99.8 & 1065 & 24 & 134 & 24 & 168 & 30 \\
\hline 01MBW801A & 15.28 & 0.18 & 9.56 & 0.01 & - & 10.51 & 1.29 & 7.74 & 0.18 & 2.26 & 0.25 & 46.72 & 0.05 & 1.69 & 3.8 & 99.52 & 1705 & 44 & 392 & 16 & 114 & 26 \\
\hline $01 M B W B 04 A$ & 13.63 & 0.05 & 0.18 & $<01$ & - & 2.53 & 4.42 & 0.51 & 0.03 & 2.17 & 0.06 & 745 & $<01$ & 0.23 & 1.35 & 99.66 & 745 & 188 & 32 & 18 & 123 & 36 \\
\hline $01 \mathrm{MBWB09 \textrm {A }}$ & 13.71 & 0.06 & 0.45 & 0.01 & - & 2.83 & 4.65 & 0.34 & 0.03 & 2.32 & 0.14 & 73.35 & $<01$ & 0.41 & 1.4 & 99.7 & 705 & 2.34 & 62 & 18 & 201 & 36 \\
\hline 01MBW813A & 16.35 & 0.1 & 6.57 & $<.01$ & - & 7.64 & 1.84 & 3.76 & 0.14 & 1.86 & 0.15 & 59.66 & 0.04 & 0.86 & 0.94 & 99.91 & 1105 & 62 & 288 & 14 & 105 & 24 \\
\hline 01MBWB16A & 14.28 & 0.07 & 1.29 & 0.01 & 二 & 1.7 & 3.42 & 0.25 & 0.04 & 2.79 & 0.1 & 74.77 & 0.02 & 0.18 & 0.71 & 99.63 & 755 & 158 & 168 & 14 & 93 & 24 \\
\hline & 16.18 & 0.11 & 2.31 & 0.01 & -- & 13 & 4.57 & 2.08 & 0.07 & 2.8 & 0.21 & 63.42 & 0.03 & 0.73 & 1 & 98.65 & 1055 & 178 & 198 & 38 & 99 & 34 \\
\hline 01ME & 16.57 & 0.08 & 3.62 & 0.01 & $=$ & 3.94 & 2.33 & 1.65 & 0.05 & 3.49 & 0.21 & 65.06 & 0.05 & 0.67 & 0.80 & 98.71 & 895 & 88 & 354 & 20 & 72 & 20 \\
\hline 01MBWB27C & 15.36 & 0.02 & 8.89 & 0.01 & 6.87 & 9.24 & 1.37 & 5.77 & 0.19 & & 0.19 & 53.08 & 0.04 & 1.28 & 1.08 & 99.52 & $E$ & - & $=$ & $=$ & - & $-m$ \\
\hline
\end{tabular}

Page 3 
Table 1. (continued)

\begin{tabular}{|c|c|c|c|c|c|c|c|c|c|c|c|c|c|c|c|c|c|c|c|c|c|c|}
\hline & $\begin{array}{c}\mathrm{Al} 2 \mathrm{O} 3 \\
\%\end{array}$ & $\begin{array}{c}8 a O \\
\%\end{array}$ & $\begin{array}{c}\mathrm{CaO} \\
\%\end{array}$ & $\begin{array}{c}\mathrm{Cr} 2 \mathrm{O} 3 \\
\%\end{array}$ & $\begin{array}{c}\mathrm{FeO} \\
\%\end{array}$ & $\begin{array}{c}\mathrm{Fe} 2 \mathrm{O}^{3} \\
\%\end{array}$ & $\begin{array}{c}\mathrm{K} 20 \\
\%\end{array}$ & $\begin{array}{c}\mathrm{MgO} \\
\%\end{array}$ & $\begin{array}{c}\mathrm{MrO} \\
\%\end{array}$ & $\begin{array}{c}\mathrm{Na2O} \\
\%\end{array}$ & $\begin{array}{c}P 205 \\
\%\end{array}$ & $\begin{array}{c}\mathrm{SiO} 2 \\
\%\end{array}$ & $\begin{array}{l}\text { Sro } \\
\%\end{array}$ & $\begin{array}{c}\text { TiO2 } \\
\% \\
\end{array}$ & $\begin{array}{c}\text { LOI } \\
\%\end{array}$ & & $\begin{array}{c}\mathrm{Ba} \\
\mathrm{ppm}\end{array}$ & $\begin{array}{l}\text { Rb } \\
\mathrm{ppm}\end{array}$ & $\begin{array}{c}\mathrm{Sr} \\
\text { pom }\end{array}$ & $\begin{array}{l}\mathrm{Nb} \\
\mathrm{ppm}\end{array}$ & $\begin{array}{c}\text { Zi } \\
\text { pom }\end{array}$ & $\begin{array}{c}\mathrm{Y} \\
\mathrm{ppm}\end{array}$ \\
\hline TMBWB34A & 15.58 & $<.01$ & 10.66 & $\angle 01$ & 7.21 & 9.64 & 0.68 & 7.11 & 0.16 & 2.84 & 0.09 & 50.3 & 0.04 & 0.86 & 1.19 & 99.15 & - & - & - & - & - & - \\
\hline & & 0.14 & 1.21 & 0.01 & $\ldots$ & 81 & 5.66 & 0.97 & & & 19 & 70.14 & 0.03 & $\overline{0.47}$ & & 8.61 & 1305 & 132 & 150 & 18 & 219 & 4 \\
\hline & & & 7.22 & $<01$ & - & 33 & 1.92 & 12 & & & 0.26 & 50.19 & 0.05 & 1.2 & & & 1065 & 60 & 382 & 16 & 159 & 28 \\
\hline & 52 & 0.07 & 6.94 & $<.01$ & - & 6.63 & 2.68 & 3.35 & 0.1 & 64 & 0.2 & 54.75 & 0.05 & 1.1 & 1.52 & 99.55 & 895 & 104 & 362 & 14 & 114 & 30 \\
\hline & 19 & 0.04 & 1.19 & 0.01 & --- & 2.16 & 3.99 & 0.28 & 0.07 & 2.74 & 0.11 & 73.13 & 0.01 & 0.22 & 0.83 & 88.97 & 545 & 194 & 108 & 16 & 90 & 34 \\
\hline & 12.17 & $<01$ & 0.53 & $<.01$ & - & $\uparrow .58$ & \begin{tabular}{|l|}
4.76 \\
\end{tabular} & 0.14 & 0.02 & 1.81 & 0.03 & 77.96 & $<.04$ & 0.18 & 0.42 & 99.6 & 265 & 240 & 40 & 22 & 129 & 28 \\
\hline & 4.73 & 0.06 & 1.73 & 0.01 & - & 2.77 & 3.84 & 0.6 & 0.05 & 2.81 & 0.1 & 71.24 & 0.02 & 0.32 & 0.76 & 9.04 & 625 & 176 & 166 & 18 & 114 & 26 \\
\hline & .07 & & 0.67 & 0.01 & - & 9.94 & 0.96 & 8.2 & 0.17 & 1.55 & 0.07 & 48.93 & 0.02 & 0.9 & 26 & 16 & 310 & 48 & 182 & 6 & 36 & 20 \\
\hline & & & 5.96 & 0.01 & - & 5.7 & 2.99 & 3.95 & 0.1 & 2.48 & 0.12 & 59.49 & 0.04 & 0.68 & 1.44 & .75 & 690 & $\$ 20$ & 232 & 14 & 117 & 24 \\
\hline & 13 & 0.01 & 7.97 & 0.01 & - & 39 & 1.61 & 5.97 & 16 & 3.34 & 0.16 & 51.75 & 0.03 & 1. & .59 & 99.42 & 300 & 60 & 238 & 18 & 141 & 36 \\
\hline & & 0.06 & 4.17 & $<.01$ & - & 72 & 2.94 & 1.67 & 09 & 2.86 & 0.16 & 64.9 & 0.04 & 0.61 & & 9.32 & 685 & 114 & 256 & 16 & 144 & 32 \\
\hline & 1.73 & $<.01$ & 0.68 & $\angle .01$ & - & 88 & 3.9 & 0.05 & U. & 3.2 & 0.11 & 74.54 & $<.01$ & 0.0 & 1.71 & 98.9 & 40 & $\$ 94$ & 12 & & 33 & 26 \\
\hline & .45 & 0.04 & 0.03 & $<01$ & - & 5.56 & 2.17 & $2 . \overline{2}$ & 0.03 & 0.73 & 0.06 & 73.54 & $<.01$ & 0.56 & 2.99 & 99.36 & 525 & 98 & 34 & 18 & 240 & 22 \\
\hline & 72 & 0 & 2.92 & $<01$ & - & 19 & 2 & 0.87 & 0.05 & 3.13 & 0.1 & 66.34 & 0.04 & 0.4 & 3.91 & 98.8 & 1290 & 100 & 348 & 14 & 198 & 36 \\
\hline & 1 & 12 & 7.49 & 0.01 & $\cdots$ & 31 & 2.27 & 5.13 & 13 & & 0.13 & 57.79 & 0.03 & $0 . B^{\prime}$ & 0.5 & 2.77 & 185 & 74 & 228 & 12 & 120 & 28 \\
\hline & & 12 & 7.37 & 0.01 & - & 7.72 & 2.12 & 4.32 & 13 & & 0.13 & 58.53 & 0.04 & 0.79 & 54 & 63 & 1235 & 72 & 264 & 12 & 102 & 24 \\
\hline & & 03 & 1.34 & & $=$ & & 06 & 11.24 & 0 & & 0.56 & 34.66 & 0.07 & 2.5 & & 9.33 & \begin{tabular}{|l|}
350 \\
\end{tabular} & 34 & 688 & 74 & 210 & 22 \\
\hline & & 0.03 & 1.2 & 0.01 & - & 04 & 1.98 & 1.24 & 07 & & 09 & 75.26 & 0.02 & 051 & 37 & 9.1 & $-\infty$ & - & - & - & -- & -- \\
\hline & & $<.01$ & 48 & & - & 66 & 0.55 & 0.9 & 01 & 34 & 0.05 & 82.52 & 0.01 & 0 & 09 & 62 & 195 & 30 & 56 & 12 & 159 & 16 \\
\hline & & 0.05 & 7.13 & 0.01 & - & 67 & $\{.83$ & 7.74 & 0.17 & 76 & 0.33 & 98 & 0.03 & 2.18 & 2.1 & .43 & 610 & 70 & 248 & 38 & 177 & 26 \\
\hline & & & 2.25 & 0.01 & 3 & 68 & 3.33 & & & .44 & 0.1 & 19 & 0.03 & 0.6 & & & - & $=$ & $=$ & $\ldots$ & - & - \\
\hline & & 0 & 3.72 & $<01$ & - & 32 & 2.87 & 56 & & 1 & .11 & 68.04 & 0.03 & 0.48 & & & - & $=$ & $E$ & - & $E$ & - \\
\hline & & 0 & 1.29 & & & 05 & 2.22 & 1.51 & & 98 & 0.07 & 70.1 & 0.03 & $\overline{0.6}$ & & & $E$ & $E$ & $\ldots$ & - & 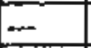 & $-m$ \\
\hline & & 0.06 & 0.2 & 0.01 & & 67 & 4.22 & 2.09 & 0.14 & 8 & 0.09 & 57.21 & 0.01 & 0.95 & 61 & 9.47 & - & - & - & - & $E$ & - \\
\hline & 4.76 & 0.07 & 3.24 & $<01$ & - & 3.53 & 2.75 & 1.17 & 0.07 & 2.87 & 0.1 & 70.18 & 0.03 & 0.4 & 0.59 & 9.76 & 720 & 156 & 156 & 16 & 123 & 26 \\
\hline & 4.65 & $<01$ & 0.67 & $<.01$ & - & 0.61 & 5.27 & 0.03 & 05 & 3.83 & 0.14 & 72.71 & $<.01$ & 0.03 & 0.3 & 98.29 & 90 & 296 & 14 & 18 & 39 & 12 \\
\hline & 18.09 & 0.08 & 0.65 & $<.01$ & 3.83 & 7.26 & 4.2 & 1.4 & 06 & 06 & 0.1 & 2.25 & 0.01 & 0.81 & 31 & 8.28 & $=$ & - & $=$ & & - & \\
\hline & & 0.1 & 7.26 & 0.01 & - & 7.73 & 2.04 & 4.67 & 13 & 1.98 & 0.14 & 8.31 & 0.04 & 0.78 & 68 & 9.37 & 1060 & 72 & 262 & 12 & 102 & 22 \\
\hline & 13 & 0.12 & 1.74 & 0.01 & $=$ & 2.15 & 3.5 & 0.4 & & 83 & 0.08 & 74.09 & 0.03 & 0.25 & 55 & 9.92 & $=$ & - & $+\infty$ & $\cdots$ & $=-\cdots$ & $\approx-$ \\
\hline & & & 09 & $<.01$ & 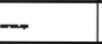 & 08 & 0.82 & & & 5.02 & 0.1 & 70.09 & $<.01$ & 0.53 & & 74 . & 265 & 38 & 76 & 26 & 180 & 30 \\
\hline & & & & & $=$ & & 0.43 & & & & 0.1 & 49.92 & 0.02 & 1.24 & 0.92 & & & it & 164 & & 72 & 28 \\
\hline & & & & 0.01 & - & & 0.84 & 7.49 & & .46 & 0.09 & 48.45 & 0.03 & 0.87 & 1.8 & & 195 & 44 & 172 & & 45 & 24 \\
\hline 01RN625A & 16.91 & $<.01$ & 14.71 & 0.01 & & 10.29 & 0.73 & 8.27 & 0.17 & 1.21 & 0.04 & $4 \overline{44} .07$ & 0.04 & 1.13 & 1.98 & 99.56 & 245 & 38 & 294 & & 57 & 26 \\
\hline
\end{tabular}


Table 1. (continued)

\begin{tabular}{|c|c|c|c|c|c|c|c|c|c|c|c|c|c|c|c|c|c|c|c|c|c|c|}
\hline SAMPLE & $\begin{array}{c}\mathrm{Al} 2 \mathrm{O} 3 \\
\%\end{array}$ & $\begin{array}{c}\mathrm{BaO} \\
\%\end{array}$ & $\begin{array}{c}\mathrm{CaO} \\
\%\end{array}$ & $\begin{array}{c}\mathrm{Cr} 203 \\
\%\end{array}$ & $\begin{array}{c}\mathrm{FeO} \\
\%\end{array}$ & $\begin{array}{c}\mathrm{Fe} 2 \mathrm{O} \\
\%\end{array}$ & $\begin{array}{c}\mathrm{K} 20 \\
\%\end{array}$ & $\begin{array}{c}\mathrm{MgO} \\
\%\end{array}$ & $\begin{array}{c}\text { MnO } \\
\%\end{array}$ & $\begin{array}{c}\mathrm{Na} 2 \mathrm{O} \\
\%\end{array}$ & $\begin{array}{c}\text { P2O5 } \\
\%\end{array}$ & $\begin{array}{c}\mathrm{SiO} 2 \\
\%\end{array}$ & $\begin{array}{c}\text { Sro } \\
\%\end{array}$ & $\begin{array}{c}\mathrm{TiO} 2 \\
\%\end{array}$ & $\begin{array}{c}\text { LOI } \\
\%\end{array}$ & TOTAL & $\begin{array}{c}\mathrm{Ba} \\
\mathrm{ppm}\end{array}$ & $\begin{array}{c}\mathrm{Rb} \\
\mathrm{ppm}\end{array}$ & $\begin{array}{c}\mathrm{Sr} \\
\mathrm{ppm}\end{array}$ & $\begin{array}{c}\mathrm{Nb} \\
\mathrm{ppm}\end{array}$ & $\begin{array}{c}\mathrm{Zr} \\
\mathrm{ppm}\end{array}$ & $\begin{array}{c}\mathrm{Y} \\
\mathrm{ppm}\end{array}$ \\
\hline $01 Z 313 \mathrm{~A}$ & 14.68 & 0.01 & 0.62 & $<.01$ & - & 1.14 & 4.53 & 0.07 & 0.03 & 2.77 & 0.18 & 74.59 & $<.01$ & \begin{tabular}{|l|}
0.1 \\
\end{tabular} & 0.91 & 99.63 & 240 & 220 & 56 & 16 & 48 & 24 \\
\hline $1 \mathrm{Z3} 32 \mathrm{~A}$ & 5.37 & 0.06 & 2.57 & $<.01$ & 3.08 & 3.86 & 3.02 & 1.88 & 0.06 & 2.78 & 0.13 & 66.69 & 0.04 & 0.56 & 1.54 & 98.56 & - & - & - & --- & 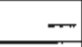 & $=$ \\
\hline $1 \mathrm{Z3} 36 \mathrm{~A}$ & 4.09 & 0.02 & 182 & 0.01 & - & 2.46 & 1.26 & 0.92 & 0.04 & 1.43 & 0.08 & 72.83 & 0.04 & 0.35 & 0.98 & 99.33 & 325 & 60 & 276 & 12 & 99 & 10 \\
\hline $012339 \mathrm{~A}$ & 16.69 & 0.03 & 4.16 & $<.01$ & - & 4.81 & 2.92 & 1.52 & 0.1 & 3.09 & 0.2 & 63.66 & 0.04 & 0.62 & 0.62 & 98.46 & 475 & 128 & 272 & 18 & 147 & 22 \\
\hline $342 \mathrm{~A}$ & 7.65 & 07 & 39 & $<.01$ & $\cdots$ & 5.95 & 2.89 & 23 & 0.09 & 2.78 & 0.23 & 59.47 & 0.05 & 0.84 & 0.72 & & 740 & 122 & 306 & 14 & 174 & 40 \\
\hline & & & 06 & & - & & 4.1 & 1.04 & .07 & & 0.13 & 67.15 & 0.03 & 0.46 & 0.6 & & 920 & 138 & 236 & 18 & 141 & 32 \\
\hline $012344 \mathrm{~A}$ & .37 & $<.01$ & 0.78 & $<.01$ & - & 0.94 & 4.73 & 0.13 & 0.04 & 3.03 & 0.12 & 75.24 & $<.01$ & 0.08 & 0.49 & 95 & 260 & 180 & 68 & 10 & 54 & 34 \\
\hline $373 \mathrm{~A}$ & 38 & 0.04 & 2.9 & & $\rightarrow$ & 3.26 & 2.96 & 0.73 & 0.08 & 2.93 & 0.12 & 70.16 & 0.02 & 0.46 & 0.48 & & 530 & 126 & 154 & 18 & 165 & 26 \\
\hline $2378 \mathrm{~A}$ & 16.44 & $<.01$ & 14.37 & 0.01 & - & 5.76 & 0.28 & 11.87 & 0.1 & 3.09 & $0 . \overline{01}$ & 47.94 & 0.01 & 0.21 & 1.56 & 65 & 35 & 16 & 128 & 6 & 15 & 10 \\
\hline $418 \mathrm{~B}$ & 4.67 & $<.04$ & 0.17 & 01 & - & 1.21 & 0.84 & 1.65 & 0.02 & 0.46 & 0.03 & 89.36 & $<.01$ & 0.14 & 1.16 & 72 & 310 & 40 & $<2$ & 8 & 96 & 12 \\
\hline & & $<01$ & 10.61 & & - & & & & & & & & 0.06 & 2.13 & 3.61 & & 70 & 14 & 518 & 16 & 126 & 24 \\
\hline $21 \mathrm{~A}$ & 87 & $<.01$ & 08 & $<01$ & - & 1.32 & 0.62 & 0.66 & 01 & 1.06 & 0.05 & 89.03 & $<.01$ & 0.19 & 3.01 & 99.9 & 185 & 42 & 22 & 12 & 144 & 14 \\
\hline $22 \mathrm{~A}$ & 9.21 & $<01$ & 0.18 & 0.01 & - & 4.45 & & & & & 0.09 & & $\leq 01$ & 0.43 & 1.66 & & 295 & 60 & 44 & 18 & 264 & 22 \\
\hline $30 \mathrm{~A}$ & 14.16 & 0.06 & 0.19 & $<.01$ & - & 6.3 & \begin{tabular}{|l|}
2.97 \\
\end{tabular} & 2.06 & 0.06 & 1.53 & 0.1 & 67.44 & 0.01 & 0.65 & 2.98 & 51 & 625 & 118 & 46 & 20 & 231 & 24 \\
\hline $2433 \mathrm{~A}$ & 13.98 & 0.15 & 4.48 & $<.01$ & - & 4.95 & 2.73 & 2.14 & 0.11 & 2.28 & 0.09 & 66.53 & 0.04 & \begin{tabular}{|l|}
0.4 \\
\end{tabular} & 0.78 & 98.66 & 1365 & 66 & 232 & 10 & 105 & 46 \\
\hline & 62 & 0.07 & 3.64 & & - & 3.9 & 2.87 & 0.94 & & & 0.11 & 69.18 & 0.02 & 0.64 & 0.61 & 9.2 & 815 & 90 & 176 & 18 & 174 & 34 \\
\hline $012439 \mathrm{~B}$ & 14.12 & $<.01$ & 0.69 & $<01$ & - & 1.05 & 4.74 & 0.15 & 0.03 & 2.54 & 0.13 & 75.38 & $<.01$ & 0.09 & 0.66 & 99.58 & 160 & 174 & 48 & 8 & 69 & 36 \\
\hline $0 \nmid Z 445 \mathrm{~A}$ & 13.33 & 0.07 & 2.08 & 0.01 & - & 3.42 & 3.58 & 1.02 & 0.06 & 2.19 & 0,09 & 71.2 & 0.03 & 0.38 & 1.2 & 66 & 750 & 150 & 188 & 20 & 153 & 36 \\
\hline $012451 \mathrm{~A}$ & 12.63 & 0.02 & 3.45 & 0.01 & -- & 4.37 & 1.29 & 0.69 & 0.08 & 2.11 & 0.11 & 73.34 & 0.05 & 0.5 & 0.74 & & 390 & 58 & 372 & 26 & 309 & 28 \\
\hline $012454 \mathrm{~A}$ & 14.61 & 0.07 & 3.25 & $<.01$ & - & 3.51 & 2.91 & 0.76 & 0.07 & 2.34 & 0.11 & 70.66 & 0.02 & 0.58 & 0.64 & & 780 & 98 & 176 & 18 & 171 & 36 \\
\hline $01 Z 459 \mathrm{~A}$ & 15.85 & 0.05 & 4.26 & 0.01 & - & 4.82 & 3.07 & 1.72 & 0.09 & 2.61 & 0.15 & 64.94 & 0.03 & 0.63 & 0.85 & 99.08 & 785 & 118 & 246 & 14 & 138 & 30 \\
\hline $012475 \mathrm{~A}$ & 14.45 & 0.06 & 0.33 & 0.01 & - & 5.22 & 3.2 & 1.55 & 0.04 & 0.85 & 0.06 & 69.36 & 0.01 & 0.64 & 2.88 & 98.66 & 735 & 126 & 122 & 18 & 204 & 32 \\
\hline 012477 & 12.36 & 0.05 & 1.13 & 0.01 & - & 4.21 & 2.48 & 1.03 & 0.06 & 1.26 & 0,1 & 73.16 & 0.02 & 0.49 & 1.87 & 98.23 & 610 & 90 & 134 & 16 & 195 & 26 \\
\hline
\end{tabular}


Table 2. Location and description of rock samples colfected for major-oxide, minor oxide, and trace element analyses in the Big Delta Quadrangle.

\begin{tabular}{|c|c|c|c|}
\hline Sample & UTM E & UTM N & Sample description \\
\hline 01JEA375A & 602764 & 7149209 & Orthogneiss \\
\hline 01JEA3B1A & 602595 & 7149637 & Orthogneiss \\
\hline 01JEA400A & 592110 & 7151118 & Dike \\
\hline 01JEA403A & 591229 & $71 \overline{51494}$ & Augen gneiss \\
\hline Q1.JEA405A & 591009 & 7151621 & Amphibolite \\
\hline 01JEA412A & 589648 & 7151746 & Othogneiss \\
\hline 01JEA424A & 587375 & 7151553 & Amphibolite \\
\hline 01 JEA432A & 602596 & 7149095 & Granodiorite \\
\hline 01JEA437A & 586540 & 7160575 & Volcanic rock \\
\hline 01JEA446A & 585667 & 7158616 & Rhyolite \\
\hline 01JEA447A & 585868 & 7158354 & Quartz monzonite \\
\hline 01JEA463A & 598044 & 7146760 & Orthogneiss \\
\hline 01JEA508A & 579089 & 7158140 & Metarhyolite \\
\hline 01JEA518A & 581365 & 7155728 & Gabbro \\
\hline 01JEA521A & 581442 & 7155534 & Metarhyolite \\
\hline 01JEA527A & 608559 & 7147829 & Orthogneiss \\
\hline 01JEA535A & 608513 & 7148665 & Orthogneiss \\
\hline 01JEA538A & 589026 & 7147979 & Amphibolite? \\
\hline 01JEA543A & 588690 & 7147300 & Amphibolite? \\
\hline 01JEA544A & 588680 & 7147234 & Plutonic rock \\
\hline 01 JEA559A & 600507 & 7147438 & Orthogneiss \\
\hline $01 \mathrm{MBW} 474 \mathrm{~A}$ & 602672 & 7149112 & Metagranodiorite \\
\hline $01 \mathrm{MgW} 477 \mathrm{~B}$ & 603132 & 7149061 & Granodiorite \\
\hline 01MBW4B6A & 603396 & 7149841 & Orthogneiss \\
\hline $01 M B W / 488 \mathrm{~A}$ & $6033 \overline{79}$ & $7\{49965$ & Augen gneiss \\
\hline 01MBW494A & $6031 \overline{12}$ & 7150437 & Igneous rock \\
\hline 01MBWA96A & 592190 & 7154919 & Metagranodiorite \\
\hline 01MBW512A & 589893 & 7154576 & Granodiorite \\
\hline 01MBW516A & 589435 & 7154480 & Augen gneiss \\
\hline 01MEW519C & 589019 & 7154552 & Metadiorite \\
\hline 01MBW528A & 592240 & 7143561 & Orthogneiss \\
\hline $01 \mathrm{MBW} 534 \mathrm{~A}$ & $5942 \overline{29}$ & 7143658 & Augen gneiss \\
\hline 01MBW541B & 596057 & 7144423 & Dike \\
\hline
\end{tabular}


Table 2. (continued)

\begin{tabular}{|c|c|c|c|}
\hline Sample & UTME & UTM N & Sample description \\
\hline $01 M B W 542 B$ & 596304 & 7144578 & Dike \\
\hline $01 M B W 585 B$ & 593446 & 7164778 & Metavolcanic \\
\hline 01MBW587A & 593469 & 7164890 & Melamafic rock \\
\hline 01MBW613A & 598446 & 7153393 & Orthogneiss \\
\hline 01MBW666A & 585532 & 7167913 & Amphibolite \\
\hline 01MBN684A & 578757 & 7161335 & Amphibolite \\
\hline 01MBV693A & 578348 & $716+951$ & Orthogneiss \\
\hline 01 MBW705A & 581318 & 7164909 & Amphibolite \\
\hline O1ABW708A & 581213 & 7164994 & Amphibolite \\
\hline $01 \mathrm{MBW} 712 \mathrm{~A}$ & 581086 & 7165184 & Amphibolite \\
\hline $01 \mathrm{MBW} 716 \mathrm{~A}$ & 581030 & 7165362 & Amphibolite \\
\hline $01 \mathrm{MBW} 720 \mathrm{C}$ & 580612 & $716555 \overline{5}$ & Amphibolite \\
\hline $01 M B W 724 \mathrm{~A}$ & $58015 \overline{2}$ & 7165857 & Amphibolite \\
\hline 01MBW732A & 579377 & $7 1 6 6 3 \longdiv { 4 }$ & Amphibolite \\
\hline $01 \mathrm{MBW} / 36 \mathrm{~A}$ & 578946 & 7166474 & Othogneiss \\
\hline $01 \mathrm{MBW} 739 \mathrm{~A}$ & 578703 & 7166419 & Amphibolite \\
\hline 01ABW740B & 578667 & 7166410 & Orthogneiss \\
\hline $01 \mathrm{MBW} 742 \mathrm{C}$ & 576582 & 7166401 & Granite \\
\hline 01MEW746A & 578258 & 7166248 & Othogneiss \\
\hline 01MBW753A & 577697 & $7+66317$ & Granite \\
\hline 01MBW/56A & 577547 & 7166314 & Amphibolite \\
\hline 01MBW765A & 605007 & 7147799 & Basaft \\
\hline 01MBW766A & 604982 & 7147833 & Tonalite \\
\hline 01MBW769A & 605027 & 7148479 & Basalt \\
\hline 01MBW799A & 586767 & 7144846 & Orthogneiss \\
\hline 01MBWB01A & 586573 & 7145686 & Gneiss \\
\hline 01MEWB04A & 586280 & 7146117 & Orthogrneiss \\
\hline 01MBW809A & 585811 & 7147407 & Augen gneiss \\
\hline 01MBWR 1 3A & 601733 & 7147839 & Diorite \\
\hline 01MBWB 16A & 601344 & 7148195 & Melagranite \\
\hline 01MBW817C & 601226 & 7148323 & Augen gneiss \\
\hline 01MBWB27B & 600576 & 7148479 & Granite \\
\hline $01 \mathrm{MBW} 27 \mathrm{C}$ & 600576 & $7 \$ \overline{48479}$ & Gnelss \\
\hline $01 \mathrm{MBW834 \textrm {A }}$ & 600168 & 7148952 & Gneiss \\
\hline
\end{tabular}


Table 2. (continued)

\begin{tabular}{|c|c|c|c|}
\hline Sample & UTME & UTM N & Samplé description \\
\hline 01MBW834B & $600+68$ & 7148952 & Augen gneiss \\
\hline 01MBW838A & 599493 & 7149510 & Dionite \\
\hline O1MBWB42A & 587961 & 7149089 & Melactionite \\
\hline 01MBW852A & 587909 & 7149730 & Plutonic rock \\
\hline 01MBW857A & 587528 & 7149768 & Augen gneiss \\
\hline $01 \mathrm{MBW} 660 \mathrm{~A}$ & 587300 & 7149930 & Melapluton \\
\hline 01MBW862A & 586866 & 7150123 & Amphibolite \\
\hline O1RN434A & 593306 & 7154872 & Granodiorite \\
\hline 01RN438A & 594061 & 7154717 & Amphibolite \\
\hline 01RN443A & 595458 & 7154702 & Monzogranite \\
\hline O1RN460B & 598297 & 7148930 & Granite \\
\hline 01RN479A & 583663 & 7163079 & Phyllite/meta-andestie? \\
\hline 01RN509C & 594969 & 7150221 & Porphyry \\
\hline 01RN511A & 596139 & 7149405 & Dionite \\
\hline 01RN516A & 596711 & 7148864 & Diorite \\
\hline 01RN522A & 582844 & 7163345 & Greenstone \\
\hline 01RN523A & 582666 & 7163367 & Metatuff \\
\hline 0)RN533A & 581784 & 7163340 & Melarhyolite \\
\hline 01RN533B & 581784 & 7163340 & Phylite/metabasite? \\
\hline 01RN534A & 567861 & 7165300 & Gneiss \\
\hline 01RN535A & 567783 & 7165508 & Orthogneiss \\
\hline 01RN538A & 568080 & 7166168 & Gneiss \\
\hline 01RN540A & 568942 & 7166399 & Schist \\
\hline 01RN546A & 572612 & 7166440 & Orthogneiss \\
\hline 01RN5468 & 572612 & 7166440 & Aplite \\
\hline O1RN551A & 573460 & 7167220 & Schist \\
\hline $01 R N 560 \mathrm{~A}$ & 608489 & 7146958 & Quartz diorile \\
\hline 01RN565A & 607316 & 7147655 & Orthogneiss \\
\hline 01RN579A & 590462 & 7144930 & Othogneiss \\
\hline 01RN605A & 605200 & 7143003 & Amphibolite \\
\hline o1RN6148 & 587388 & 7148134 & Amphibolite \\
\hline O1RN625A & 590034 & 7148120 & Amphibolile \\
\hline
\end{tabular}


Table 2. continued

\begin{tabular}{|c|c|c|c|}
\hline Sample & UTM E & UTM N & Sample description \\
\hline $012313 \mathrm{~A}$ & 600244 & 7149834 & Granodiorite \\
\hline $012332 \mathrm{~A}$ & 595529 & 7152930 & Gneiss/orthogneiss? \\
\hline $012336 \mathrm{~B}$ & 596508 & 7152449 & Aplite \\
\hline $012339 \mathrm{~A}$ & 597098 & 7152595 & Monzodiorite \\
\hline $012342 A$ & 598011 & $71523 B i$ & Granodiorite \\
\hline $01 Z 343 \mathrm{~A}$ & 598194 & 7152337 & Granodiorite \\
\hline $012344 \mathrm{~A}$ & 598564 & 7152251 & Granodiorite \\
\hline $01 Z 373 \mathrm{~A}$ & 601495 & 7145927 & Tonalite \\
\hline $01 Z 378 \mathrm{~A}$ & 599984 & 7145364 & Amphibolite \\
\hline $01 Z 418 \mathrm{~B}$ & 580907 & 7157316 & Metatuff \\
\hline $012418 \mathrm{C}$ & 580907 & 7157316 & Greenstone \\
\hline $012421 \mathrm{~A}$ & 581183 & 7157077 & Metatuff \\
\hline $01 Z 422 \mathrm{~A}$ & 581229 & 7156988 & Greenstone \\
\hline $01 Z 430 \mathrm{~A}$ & 580270 & 7156121 & Greenstone \\
\hline $01 Z 433 \mathrm{~A}$ & 608540 & 7147596 & Orthogneiss \\
\hline 01Z439A & 608003 & 7148838 & Quartz diorite \\
\hline $01 Z 4398$ & 608003 & 7148838 & Granite \\
\hline $01 Z 445 \mathrm{~A}$ & 593855 & 7144643 & Orthogneiss/meta-pluton? \\
\hline $012451 \mathrm{~A}$ & 594013 & 7146096 & Gneiss \\
\hline $01 Z 454 \mathrm{~A}$ & 607633 & $7 \longdiv { 4 9 3 2 2 }$ & Quartz diorite \\
\hline $012459 \mathrm{~A}$ & 608226 & $714 \overline{49769}$ & Granodiorite \\
\hline $012475 \mathrm{~A}$ & 601875 & 7151882 & Orthogneiss \\
\hline $012477 \mathrm{~A}$ & 601544 & 7152070 & Orthogneiss \\
\hline
\end{tabular}


Table 3. Concentration of trace elements in rock samples collected in the Big Delta quadrangle.

\begin{tabular}{|c|c|c|c|c|c|c|c|c|c|c|c|c|c|c|c|c|c|c|c|c|}
\hline SAMPLE & $\begin{array}{c}\mathrm{Au} \\
\mathrm{ppb}\end{array}$ & $\begin{array}{l}\mathrm{Au} \\
\mathrm{ppb}\end{array}$ & $\begin{array}{c}\mathrm{Pt} \\
\mathrm{ppb}\end{array}$ & $\begin{array}{l}P d \\
\mathrm{ppb}\end{array}$ & $\begin{array}{c}\mathrm{Ag} \\
\mathrm{ppm}\end{array}$ & $\begin{array}{l}\mathrm{Al} \\
\%\end{array}$ & $\begin{array}{c}\text { As } \\
\mathrm{ppm}\end{array}$ & $\begin{array}{c} \\
\text { ppm }\end{array}$ & $\begin{array}{c}\mathrm{Ba} \\
\mathrm{ppm}\end{array}$ & $\begin{array}{c}\text { Be } \\
\text { opm }\end{array}$ & $\begin{array}{c}\mathrm{Bi} \\
\mathrm{ppm}\end{array}$ & $\begin{array}{l}\mathrm{Ca} \\
\%\end{array}$ & $\begin{array}{c}\mathrm{Cd} \\
\mathrm{ppm}\end{array}$ & $\begin{array}{c}\text { Co } \\
\text { ppm }\end{array}$ & $\begin{array}{c}\mathrm{Cr} \\
\mathrm{ppm}\end{array}$ & $\begin{array}{l}\mathrm{Cu} \\
\mathrm{ppm}\end{array}$ & $\begin{array}{l}\mathrm{Fe} \\
\%\end{array}$ & $\begin{array}{c}\mathrm{Ga} \\
\text { ppm }\end{array}$ & $\begin{array}{c}\mathrm{Hg} \\
\mathrm{ppm}\end{array}$ & $\begin{array}{l}K \\
\%\end{array}$ \\
\hline $01 \mathrm{JEA} 3 \overline{7} \bar{A}$ & $<5$ & -- & - & - & $<.2$ & 2.12 & 28 & 10 & 110 & $<.5$ & 6 & 0.07 & $<5$ & 7 & 148 & 14 & 3.45 & 10 & $<1$ & $\overline{1.17}$ \\
\hline $01 \mathrm{JEA394A}$ & 150 & -- & - & $\cdots$ & $<.2$ & 0.26 & $>10000$ & $<10$ & 220 & $<.5$ & $<2$ & 0.04 & 0.5 & 3 & 142 & 5 & 1.81 & $<10$ & 1 & 0.21 \\
\hline 01JEA403B & $<5$ & -- & - & - & $<.2$ & 8.3 & 44 & $<10$ & 80 & 3.5 & $<2$ & 5.55 & $<.5$ & 2 & 73 & 19 & 0.98 & 30 & 3 & 0.08 \\
\hline $01 \mathrm{JEA551 \textrm {A }}$ & 45 & - & - & - & $<2$ & 2.71 & 254 & 10 & 150 & $<.5$ & $<2$ & 0.59 & $<.5$ & 13 & 133 & 15 & \begin{tabular}{|l|}
3.82 \\
\end{tabular} & 30 & 7 & 0.27 \\
\hline $01 \mathrm{MBW} 476 \mathrm{~A}$ & 10 & - & - & - & 0.2 & $0.4 \overline{5}$ & 8 & $<10$ & 30 & $<.5$ & $<2$ & 0.03 & 0.5 & 1 & 186 & 13 & 0.95 & 10 & $<1$ & 0.16 \\
\hline $01 \mathrm{MBW} / 47 \overline{\mathrm{A}}$ & 20 & - & $\cdots$ & - & $<.2$ & 0.34 & $<2$ & $<10$ & 10 & $<\overline{5}$ & 10 & 0.29 & $<.5$ & $<1$ & 99 & 11 & 0.58 & $<10$ & $<1$ & 0.15 \\
\hline 01MBW/42A & $<5$ & - & - & -- & 0.2 & 0.29 & 8 & $<10$ & $<10$ & $<.5$ & $<2$ & 0.33 & $<.5$ & 2 & 171 & 539 & 0.41 & $<10$ & 4 & 0.03 \\
\hline $01 \mathrm{MBW} 501 \mathrm{~A}$ & 105 & - & - & - & $<.2$ & 6.46 & $<2$ & $<10$ & 150 & 1.5 & $<2$ & 4.24 & $\overline{<.5}$ & 8 & 65 & 43 & 1.43 & 30 & $<1$ & 0.05 \\
\hline 01MBW519B & $<5$ & - & - & - & $<.2$ & 0.03 & $<2$ & $<10$ & $<10$ & $<.5$ & $<2$ & 0.01 & $<.5$ & $<1$ & 236 & 8 & 0.31 & $<10$ & $<1$ & 0.01 \\
\hline $01 \mathrm{MBW} 531 \mathrm{~B}$ & $<5$ & $\cdots$ & $\cdots$ & - & $<.2$ & 0.22 & 2 & 10 & 10 & $<.5$ & $<2$ & 0.02 & 0.5 & $<1$ & 164 & 6 & 0.53 & 10 & 1 & 0.07 \\
\hline $01 \overline{\mathrm{MBW}}$ 534B & $<5$ & -- & - & - & $<.2$ & 0.01 & 2 & 10 & $<10$ & $<.5$ & 8 & 0.01 & $<.5$ & 1 & 214 & 10 & 0.38 & $<10$ & $<1$ & $<.01$ \\
\hline $01 \overline{M B W 535 B}$ & $<5$ & -- & - & -- & $<.2$ & 0.58 & 2 & $<10$ & 40 & $<.5$ & $<2$ & 0.03 & $<.5$ & 3 & 126 & 8 & 1.11 & 10 & $<1$ & 0.12 \\
\hline 01MBW541C & $<5$ & -- & -- & - & $<.2$ & 0.15 & $<2$ & $<10$ & $<10$ & $<\overline{5}$ & 6 & $<.01$ & $<.5$ & 8 & 174 & 36 & $\overline{0.76}$ & 10 & $<1$ & 0.02 \\
\hline 01MBW552A & $<5$ & - & - & -- & $<.2$ & 0.44 & 10 & $<10$ & 10 & $<\overline{5}$ & $<2$ & 0.02 & $<.5$ & 1 & 90 & 10 & 0.87 & 10 & $<1$ & 0.06 \\
\hline $01 \mathrm{MBW} 560 \mathrm{C}$ & $<5$ & - & - & $\cdots$ & $<.2$ & 0.24 & $<2$ & $<10$ & $<10$ & $<5$ & 6 & 0.01 & $<.5$ & 1 & 156 & 7 & 0.59 & $<10$ & 1 & 0.01 \\
\hline 01MBW567B & $<5$ & $\cdots$ & $\cdots$ & $\ldots$ & 0.2 & 0.15 & 4 & $<10$ & $<10$ & $<.5$ & 6 & $<.01$ & 1 & 1 & 225 & 19 & \begin{tabular}{|l|}
1.41 \\
\end{tabular} & 10 & $<1$ & $<.01$ \\
\hline $01 \mathrm{MB}$ & $<5$ & - & - & -- & $<.2$ & 0.27 & 6 & $<10$ & 10 & $<5$ & 6 & 0.04 & $<.5$ & 1 & 122 & 8 & $\overline{0.96}$ & $<10$ & 3 & 0.15 \\
\hline 01MBW668A & $<5$ & - & $=$ & --- & $<.2$ & 2.28 & 10 & 10 & 60 & $0 . \overline{5}$ & 2 & 0.32 & $<5$ & 13 & 93 & 42 & 3.6 & 20 & 1 & 0.25 \\
\hline 01MBW670A & $<5$ & - & - & -- & $<.2$ & 0.07 & $<2$ & $<10$ & $<10$ & $<.5$ & 8 & $<.01$ & $<.5$ & 11 & $14 B$ & 105 & 2.37 & $<10$ & 3 & 0.02 \\
\hline $01 \mathrm{MB}$ & $\ldots$ & 1 & 23 & 17 & $<2$ & 0.37 & $<2$ & 10 & $<10$ & $<5$ & $<2$ & 0.78 & $<.5$ & 41 & 633 & 47 & 2.58 & 10 & $<1$ & $<.01$ \\
\hline $01 \mathrm{MBW} 707 \mathrm{C}$ & $<5$ & - & $\ldots$ & - & $<.2$ & 0.14 & 12 & $<10$ & 20 & $<.5$ & $<2$ & 0.04 & $<.5$ & 3 & 196 & 37 & 1.13 & $<10$ & 3 & 0.06 \\
\hline $01 \overline{M B W 760 \mathrm{~A}}$ & - & $<1$ & 8.5 & 9 & $<.2$ & 3.77 & $<2$ & $<10$ & 10 & $<.5$ & $<2$ & 2.67 & $<.5$ & 5 & 82 & 43 & 0.58 & 10 & $<1$ & 0.07 \\
\hline $01 \overline{\mathrm{MBV}}$ & - & $<1$ & 2.5 & 1 & $<.2$ & 0.36 & $<2$ & 10 & $<10$ & $<.5$ & $<2$ & 0.07 & $<.5$ & 42 & 416 & 9 & 2.53 & 10 & $<1$ & $<.01$ \\
\hline $01 \mathrm{MBW} 760 \mathrm{E}$ & -- & $<1$ & 3.5 & $<1$ & $<.2$ & 0.72 & $<2$ & $<10$ & $<10$ & $<.5$ & $<2$ & 0.08 & 4.5 & 9 & 565 & 8 & 0.66 & 10 & $<1$ & $<.01$ \\
\hline 01MBW761A & $<5$ & - & $\cdots$ & -- & $<.2$ & 2.77 & $<2$ & 10 & 200 & $<\overline{5}$ & 6 & 1.24 & $<5$ & 12 & 117 & 10 & 3.17 & 10 & $<1$ & 0.36 \\
\hline $01 \mathrm{MBW} 768 \mathrm{~A}$ & $<5$ & - & - & --- & 0.2 & 2.53 & 2 & 10 & 650 & $<.5$ & 8 & 0.81 & $<.5$ & 5 & 71 & 1 & 3.19 & $<10$ & $<1$ & 0.81 \\
\hline 01MBW792B & $<5$ & - & - & -- & $<.2$ & 0.17 & $<2$ & $<10$ & $<10$ & $<.5$ & 8 & 0.06 & $<.5$ & $<1$ & 136 & $<1$ & 0.27 & $<10$ & $<1$ & 0.03 \\
\hline 01MBW793B & 5 & - & $\cdots$ & - & 0.2 & 0.16 & $<2$ & 40 & $<10$ & $<.5$ & 2 & 0.12 & $<.5$ & $<1$ & 64 & $<1$ & 01 & $<10$ & 1 & 0.01 \\
\hline 01MBW811A & $<5$ & - & - & - & $<2$ & 0.07 & $<2$ & 10 & $<10$ & $<.5$ & 2 & $<01$ & $<.5$ & 1 & 182 & $<1$ & 0.38 & $<10$ & 1 & 0.02 \\
\hline 01MBW817日 & 510 & $\cdots$ & $=$ & - & 1 & 0.66 & 726 & $<10$ & 70 & $<.5$ & 12 & 0.05 & $<.5$ & 1 & 48 & 40 & 1.39 & $<10$ & $<1$ & 0.27 \\
\hline $01 \mathrm{MBW826 \textrm {C }}$ & 490 & - & $\cdots$ & - & 0.2 & 5.06 & 16 & 10 & $<10$ & $\overline{2}$ & 8 & 4.67 & $<.5$ & 6 & 93 & 35 & 1.01 & 10 & $<1$ & 0.03 \\
\hline 01MBW828B & 510 & -- & -- & - & 1 & 1.64 & 832 & $<10$ & $<10$ & 1 & 10 & 1.27 & $<.5$ & 16 & 96 & 211 & 3.85 & $<10$ & $<1$ & $<.01$ \\
\hline $01 \mathrm{MBW} 839 \mathrm{~A}$ & 35 & - & - & $=$ & 0.2 & 0.43 & 84 & 10 & 10 & $<5$ & $<2$ & 0.47 & $<.5$ & 2 & 163 & 3 & 0.55 & $<10$ & 1 & 0.04 \\
\hline O1MBW848A & $<5$ & - & $\cdots$ & - & 0.2 & 0.69 & 4 & 10 & 50 & $<.5$ & 6 & 0.3 & $<.5$ & 4 & 126 & 82 & 2.16 & $<10$ & $<1$ & 0.25 \\
\hline $01 \mathrm{MBV}$ & $<5$ & - & -- & -- & 0.2 & 0.39 & $<2$ & $<10$ & $<10$ & $<.5$ & 8 & 0.01 & $<.5$ & 2 & 108 & 4 & 0.59 & $<10$ & $<1$ & 0.14 \\
\hline $01 M B W 850 \mathrm{~A}$ & $<5$ & - & $-\cdots$ & $\cdots$ & 0.2 & 0.49 & $<2$ & $<10$ & 10 & $<5$ & 4 & 0.06 & $<.5$ & 1 & 63 & $<1$ & 0.88 & $<10$ & $<1$ & 0.14 \\
\hline $01 \mathrm{RN} 460 \mathrm{~B}$ & 5 & $-\infty$ & -- & 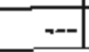 & 0.2 & 0.32 & $\overline{36}$ & $<10$ & $<10$ & 0.5 & 14 & 0.04 & $<.5$ & $<1$ & 91 & $<1$ & 0.53 & $<10$ & 3 & $\overline{0.18}$ \\
\hline
\end{tabular}

Page 10 
듬

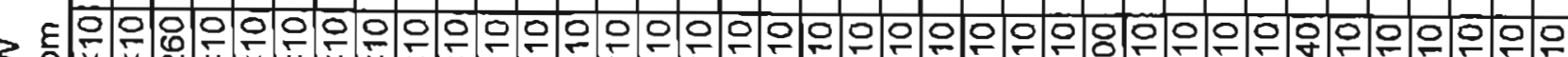

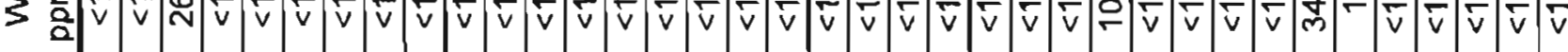

$>$

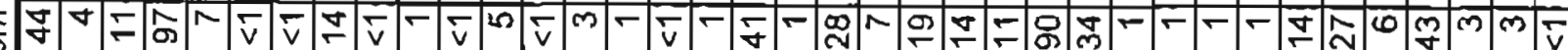

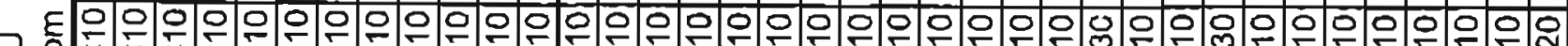

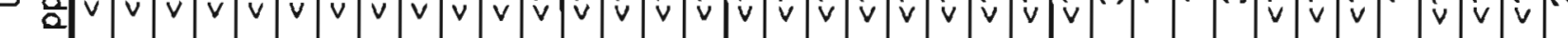

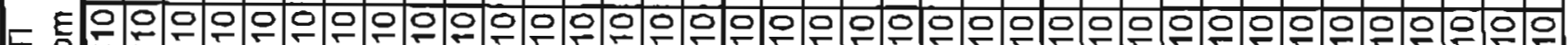

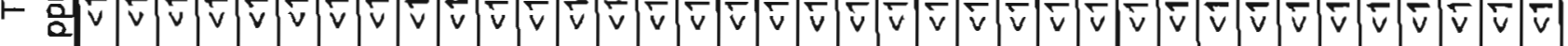

i= 웅

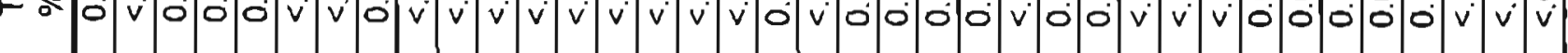

ふ্ড

ह

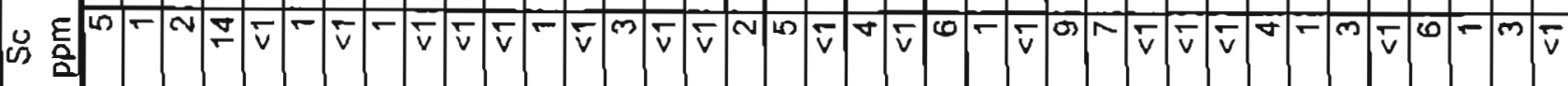

ले

๑

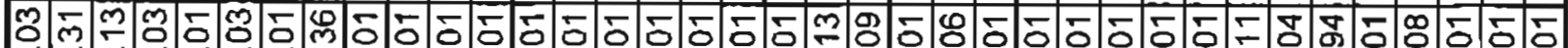

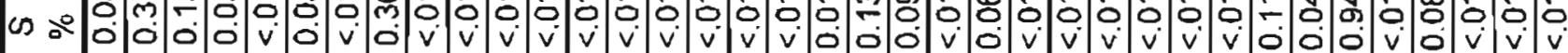

a

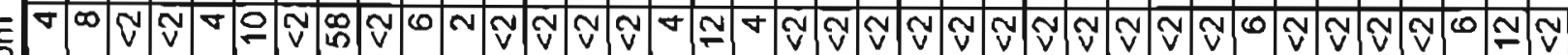

定

a.

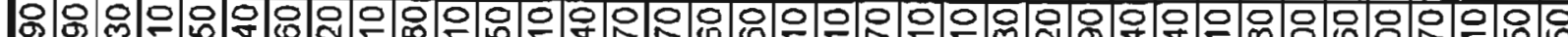

a

iz

E

« M-

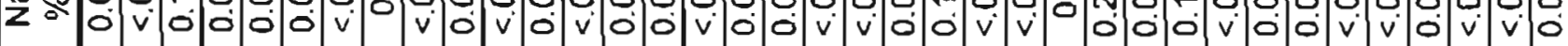

造

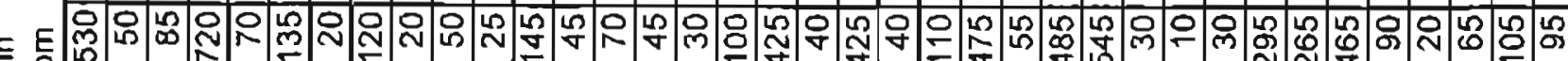

歪

प \&

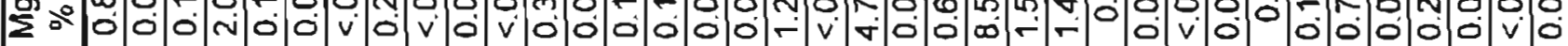

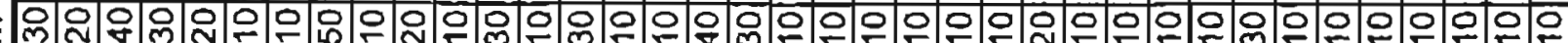

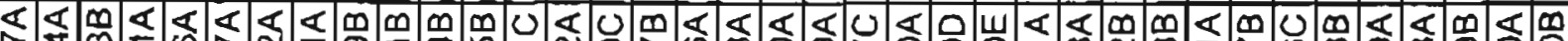

స్రార్ర

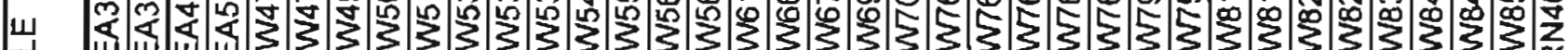

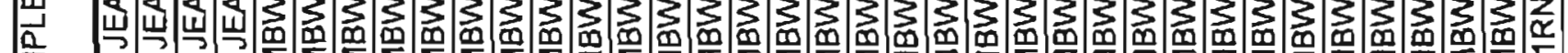

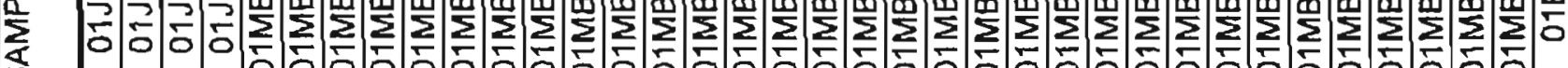


× ஃ

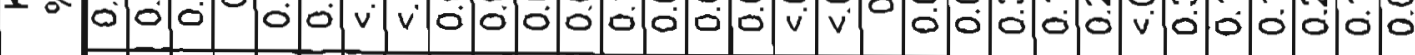

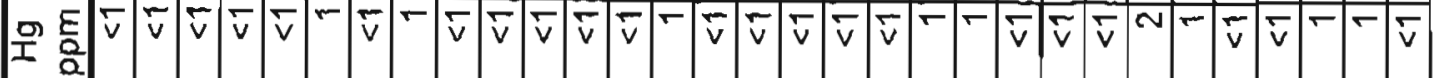

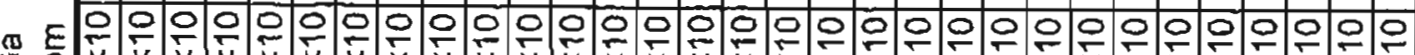

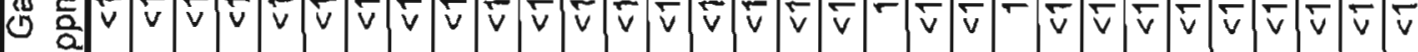

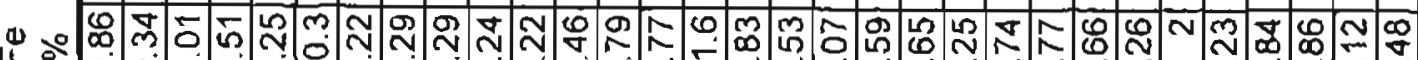

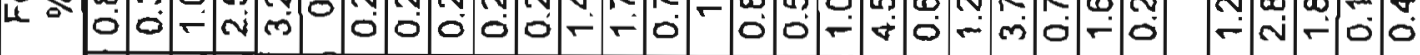

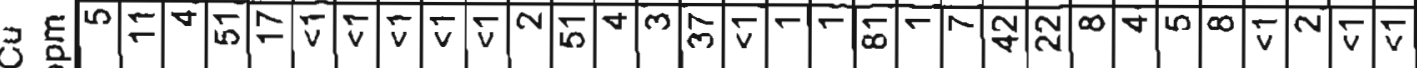

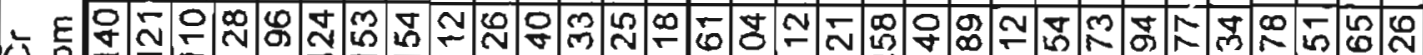

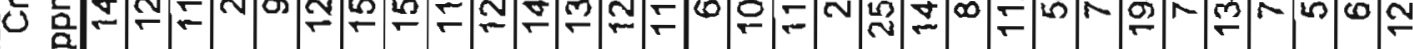
8 हn की

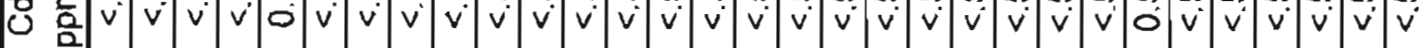
ษ a

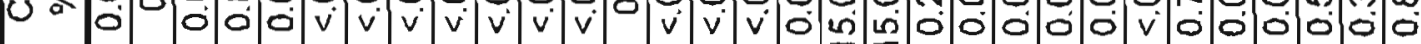
酉

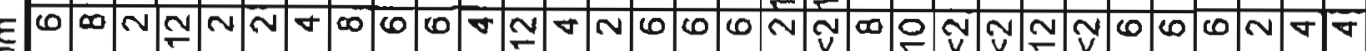
西

凹

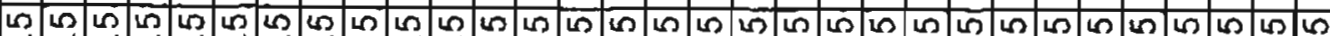
$\infty$

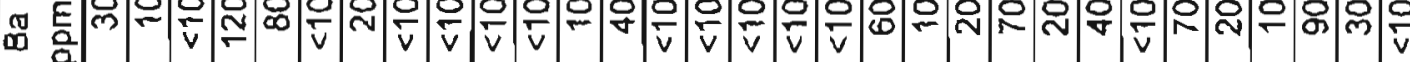

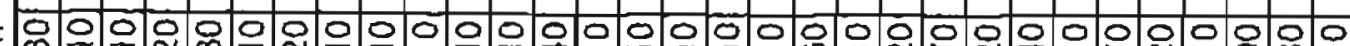
$\infty$

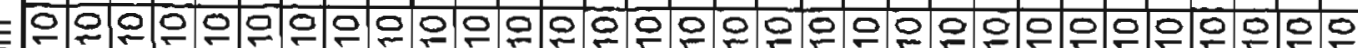

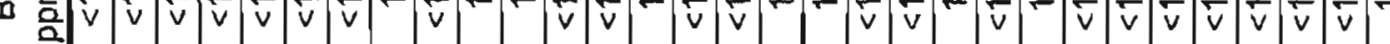
电

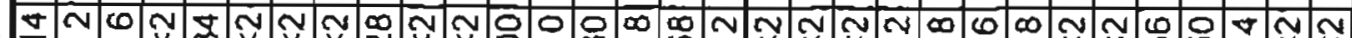
$<$

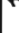

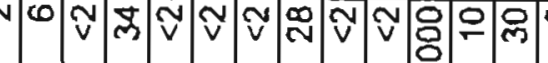

MN

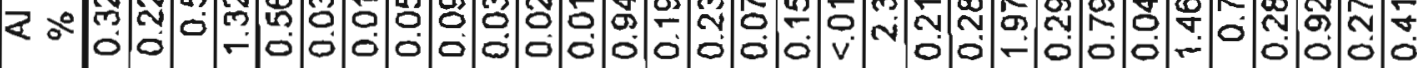
NNNNM

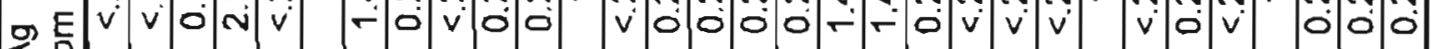

뭉 응

芒 융 군 응

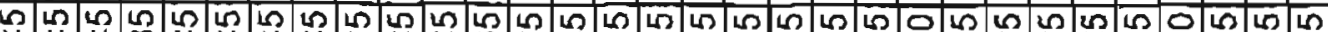
$<$ 이

m

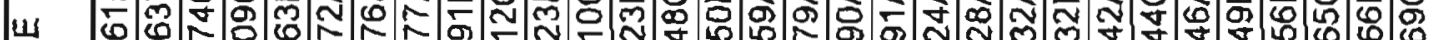
U

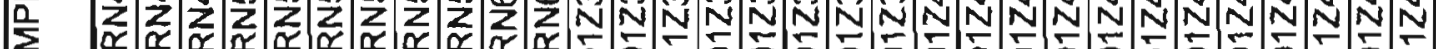

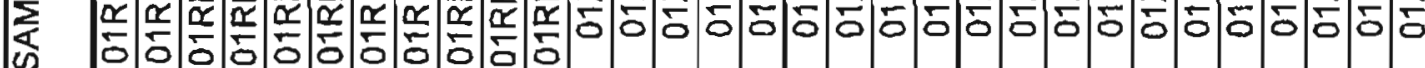




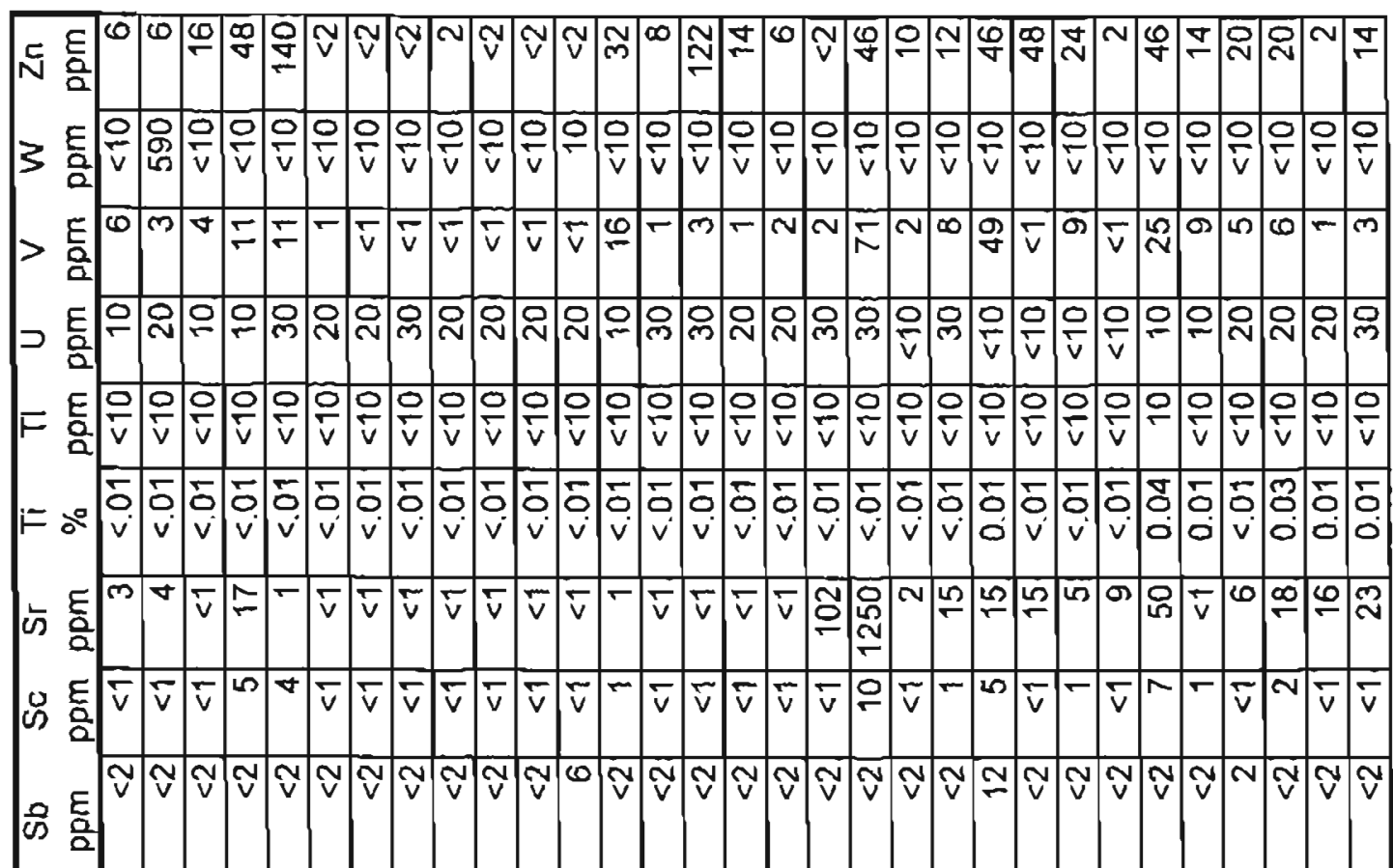

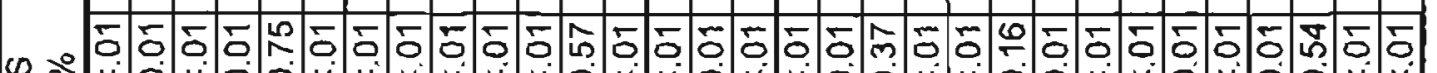

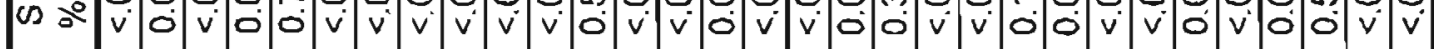

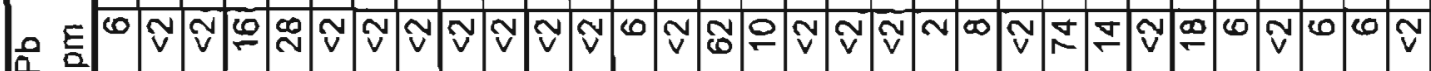
送

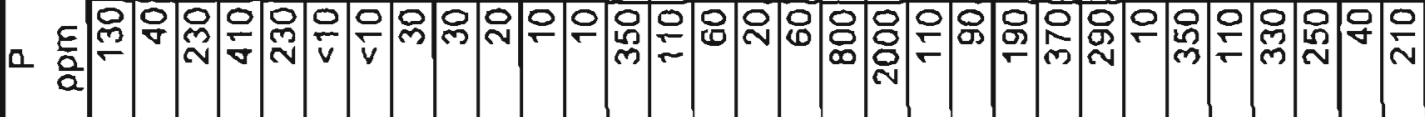
$\bar{z}$

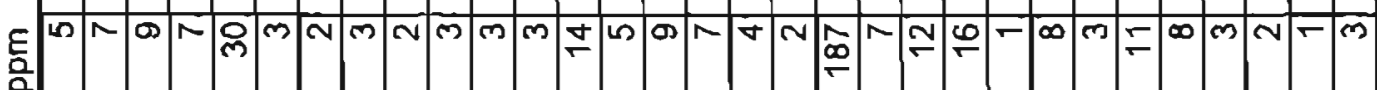
س

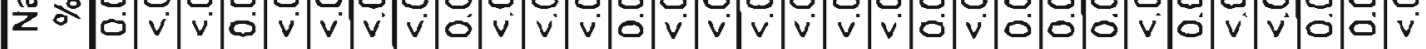

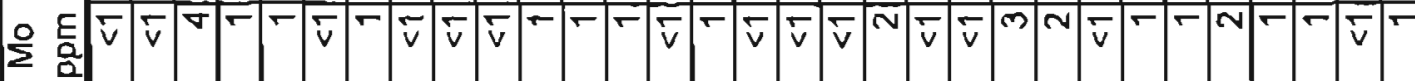
든

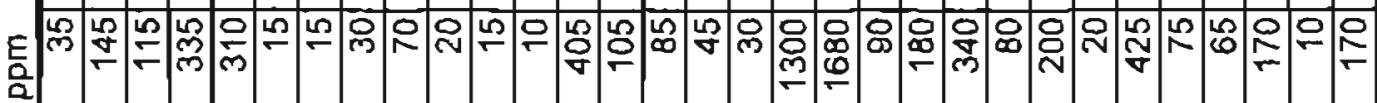

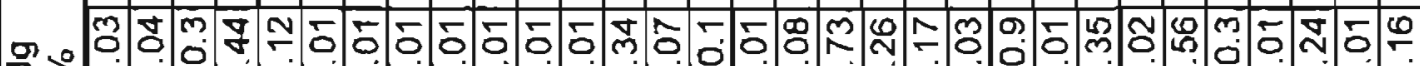

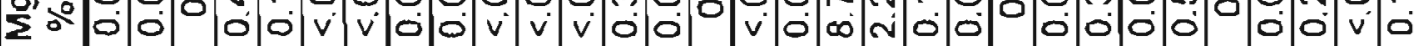

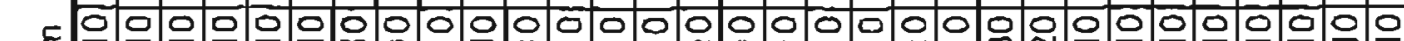

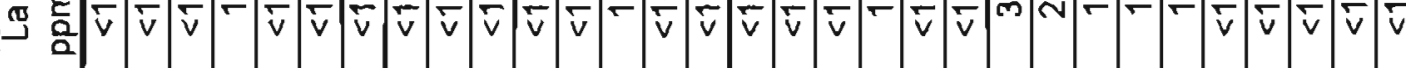
m

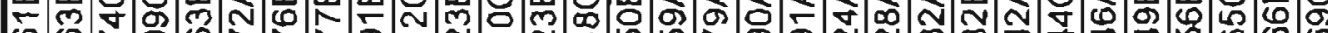

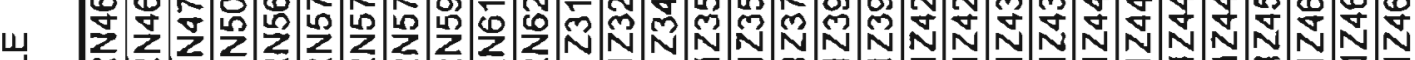

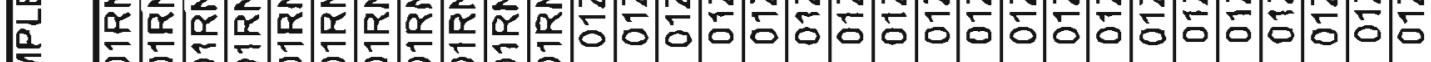


Table 4. Location and description of rock samples collected in the Big Delta quadrangle for trace element geochemical analyses.

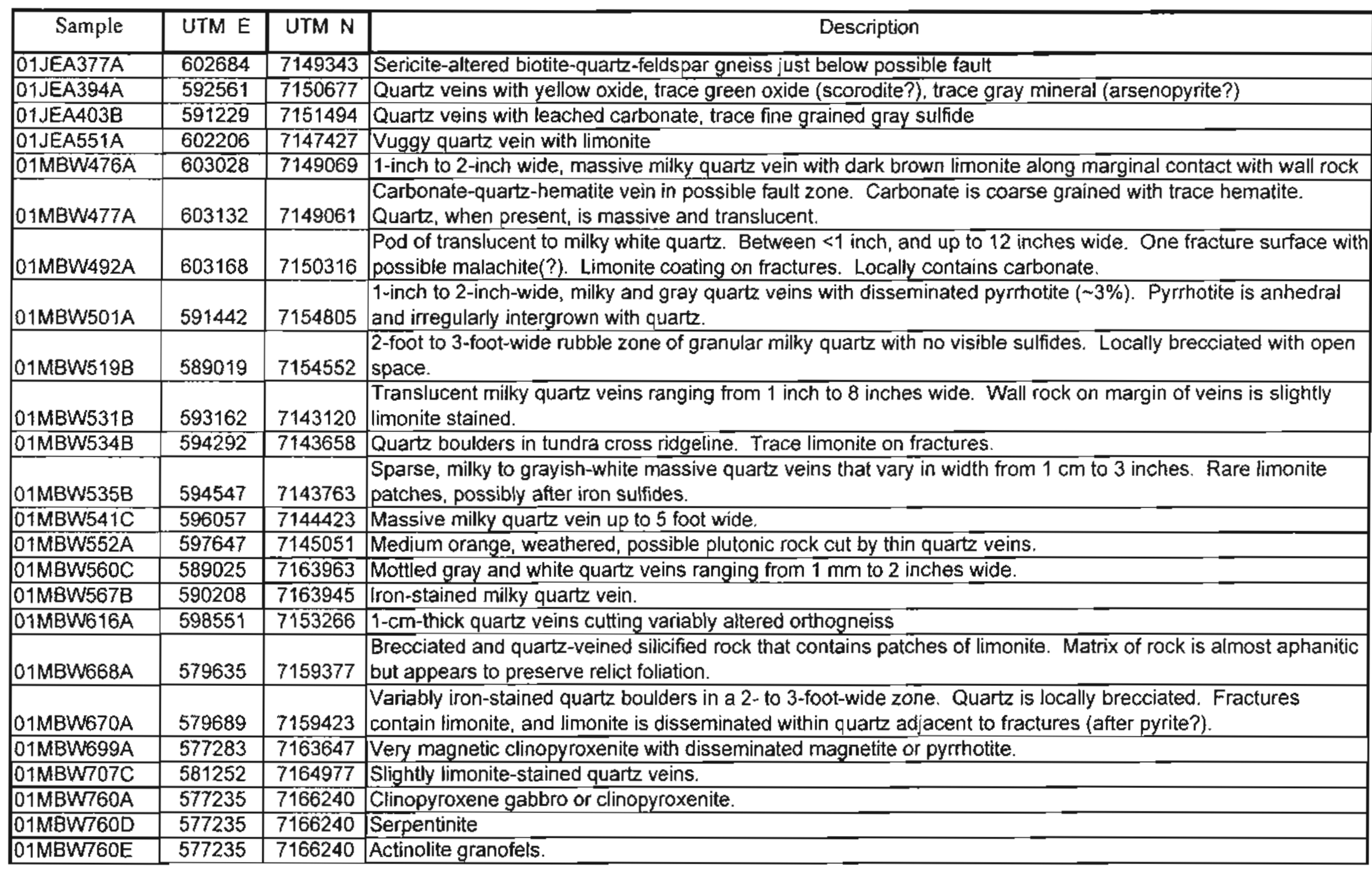




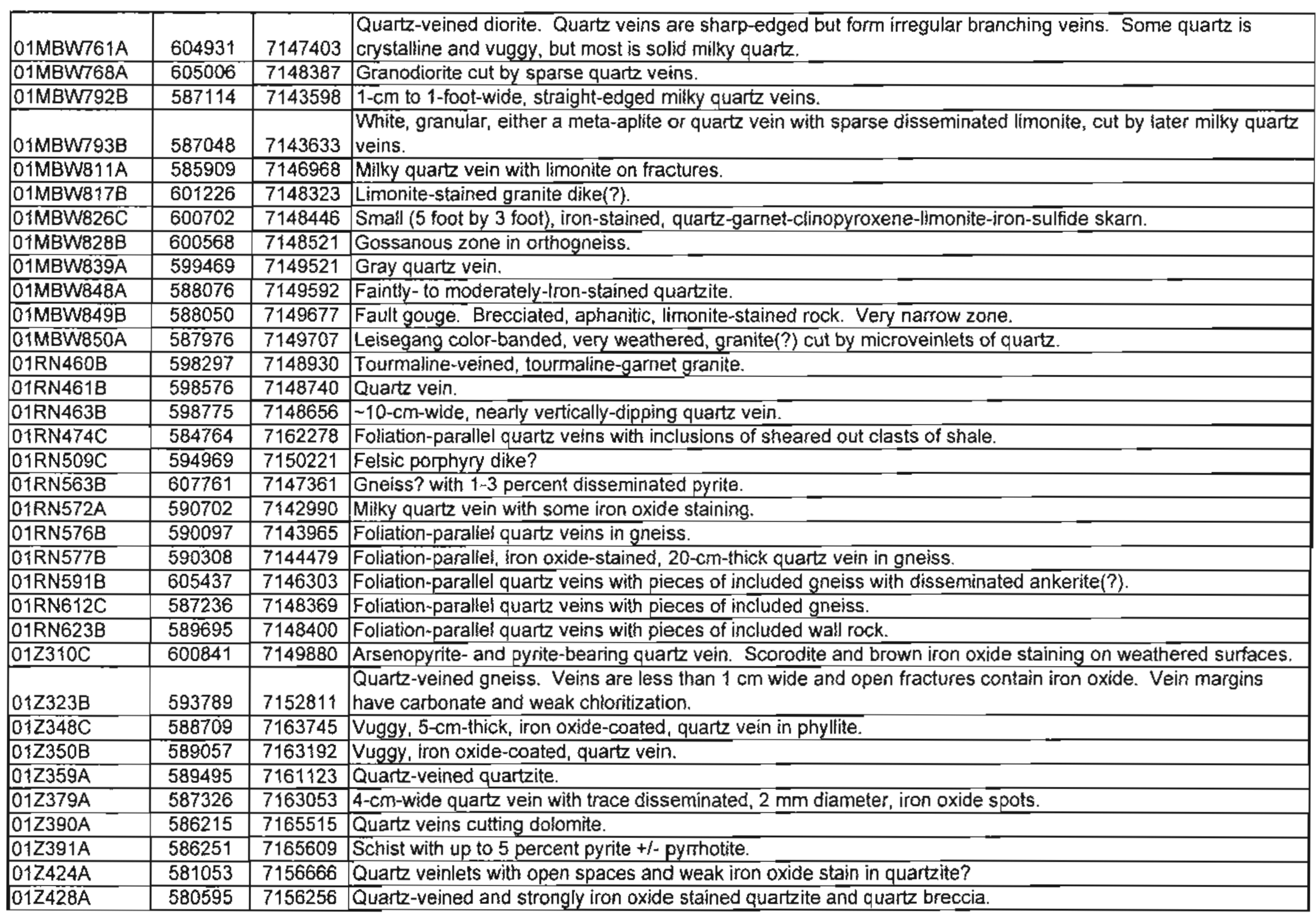




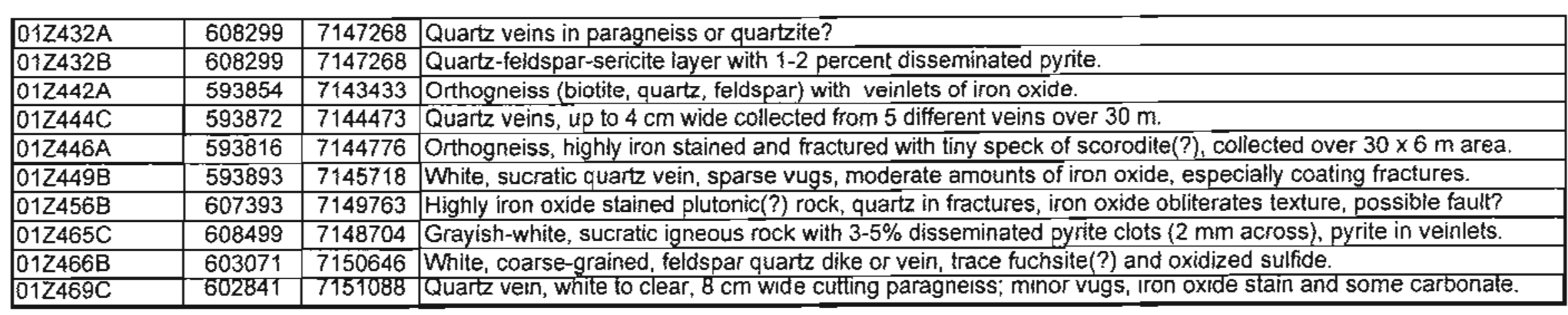


Table 5. Limits and analytical methods for major oxide, minor oxide and trace element analyses. Analytical method XRF $=X$-Ray Fluorescence Spectroscopy. Note: $\mathrm{LOI}^{*}=$ Loss on Ignition. Total ${ }^{*}=$ Total percent as oxide.

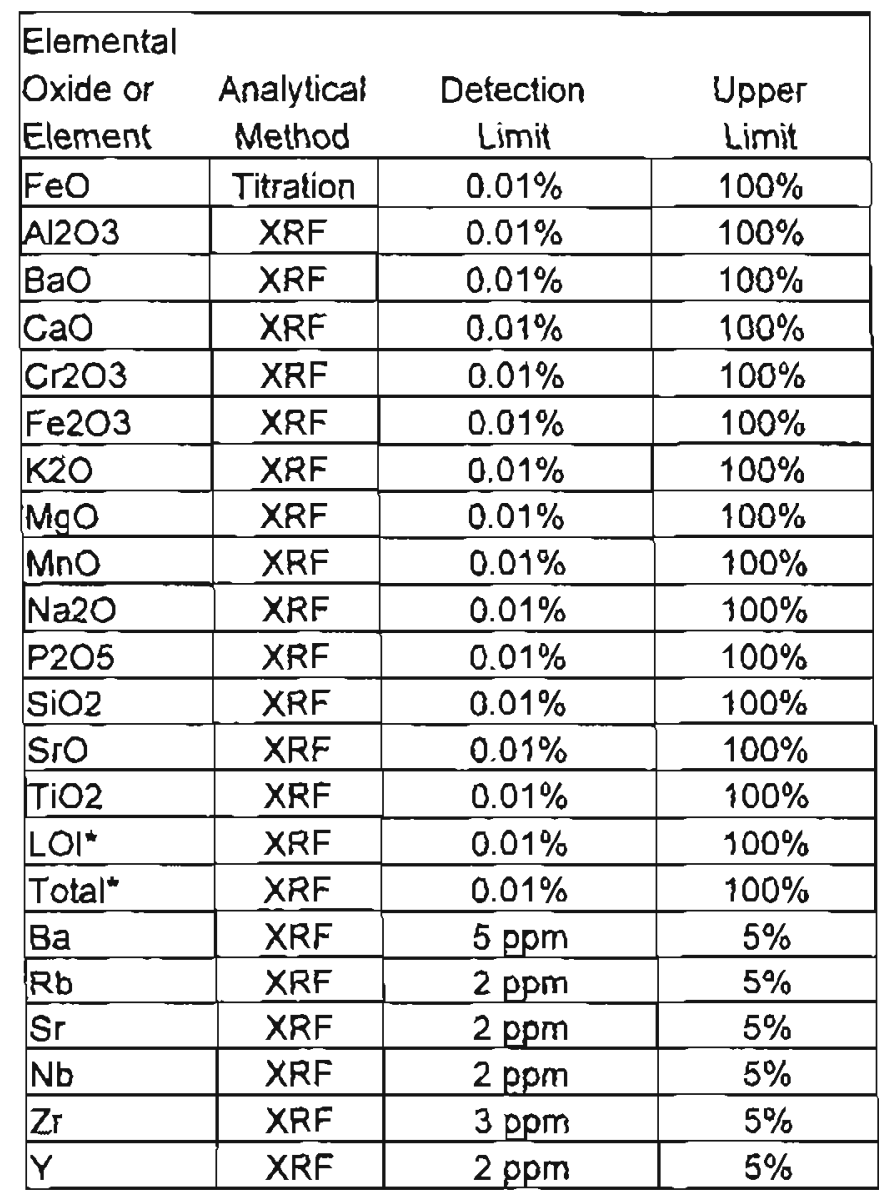


Table 6. Limits and analytical methods for trace element geochemical analyses. Analytical methods include: FA-AAS = Fire Assay-Atomic Absorption Spectroscopy, ICP-MS = Inductively Coupled Plasma-Mass Spectroscopy, ICPAES = Inductively Coupled Plasma-Atomic Emission Spectroscopy

\begin{tabular}{|c|c|c|c|c|}
\hline Element & \begin{tabular}{|c} 
Possible \\
Incomplete \\
Digestion
\end{tabular} & $\begin{array}{c}\text { Analytical } \\
\text { Method }\end{array}$ & $\begin{array}{c}\text { Detection } \\
\text { Limit }\end{array}$ & $\begin{array}{l}\text { Upper } \\
\text { Limit }\end{array}$ \\
\hline $\mathrm{Au}$ & & FA-AAS & $5 \mathrm{ppb}$ & $10000 \%$ \\
\hline $\mathrm{Au}$ & & ICP-MS & $1 \mathrm{ppb}$ & $1000 \mathrm{ppb}$ \\
\hline $\mathrm{Pt}$ & & ICP-MS & $0.5 \mathrm{ppb}$ & $1000 \mathrm{ppb}$ \\
\hline $\mathrm{Pd}$ & & ICP-MS & $1 \mathrm{ppb}$ & $1000 \mathrm{ppb}$ \\
\hline $\mathrm{Ag}$ & & ICP-AES & $0.2 \mathrm{ppm}$ & $0.01 \%$ \\
\hline $\mathrm{Al}$ & 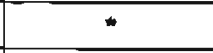 & ICP-AES & $0.01 \%$ & $15 \%$ \\
\hline As & & ICP-AES & $2 \mathrm{ppm}$ & $1 \%$ \\
\hline$B$ & & ICP-AES & $10 \mathrm{ppm}$ & $10000 \mathrm{ppm}$ \\
\hline $\mathrm{Ba}$ & $*$ & ICP-AES & $10 \mathrm{ppm}$ & $1 \%$ \\
\hline $\mathrm{Be}$ & * & ICP-AES & $0.5 \mathrm{ppm}$ & $0.01 \%$ \\
\hline $\mathrm{Bi}$ & & ICP-AES & $2 \mathrm{ppm}$ & $1 \%$ \\
\hline $\mathrm{Ca}$ & 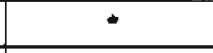 & ICP-AES & $0.01 \%$ & $15 \%$ \\
\hline $\mathrm{Cd}$ & & ICP-AES & $0.5 \mathrm{ppm}$ & $0.05 \%$ \\
\hline Co & & ICP-AES & $1 \mathrm{ppm}$ & $1 \%$ \\
\hline $\mathrm{Cr}$ & * & ICP-AES & $1 \mathrm{ppm}$ & $1 \%$ \\
\hline $\mathrm{Cu}$ & & ICP-AES & $1 \mathrm{ppm}$ & $1 \%$ \\
\hline $\mathrm{Fe}$ & & ICP-AES & $0.01 \%$ & $15 \%$ \\
\hline $\mathrm{Ga}$ & 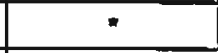 & ICP-AES & $10 \mathrm{ppm}$ & $1 \%$ \\
\hline $\mathrm{Hg}$ & & ICP-AES & $1 \mathrm{ppm}$ & $1 \%$ \\
\hline $\mathrm{K}$ & 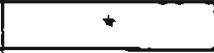 & ICP-AES & $0.01 \%$ & $0.01 \%$ \\
\hline $\mathrm{La}$ & $*$ & ICP-AES & $10 \mathrm{ppm}$ & $1 \%$ \\
\hline $\mathrm{Mg}$ & 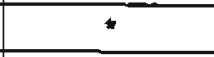 & ICP-AES & $0.01 \%$ & $15 \%$ \\
\hline $\mathrm{Mn}$ & & ICP-AES & $5 \mathrm{ppm}$ & $1 \%$ \\
\hline Mo & & ICP-AES & $1 \mathrm{ppm}$ & $1 \%$ \\
\hline $\mathrm{Na}$ & $\star$ & ICP-AES & $0.01 \%$ & $10 \%$ \\
\hline $\mathrm{Ni}$ & & ICP-AES & $1 \mathrm{ppm}$ & $1 \%$ \\
\hline $\mathrm{P}$ & & ICP-AES & $10 \mathrm{ppm}$ & $1 \%$ \\
\hline$P b$ & & ICP-AES & $2 \mathrm{ppm}$ & $1 \%$ \\
\hline$s$ & & ICP-AES & $0.01 \%$ & $10 \%$ \\
\hline Sb & & ICP-AES & $2 \mathrm{ppm}$ & $1 \%$ \\
\hline Sc & 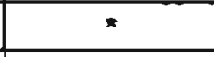 & ICP-AES & $1 \mathrm{ppm}$ & $1 \%$ \\
\hline $\mathrm{sr}$ & * & ICP-AES & $1 \mathrm{ppm}$ & $1 \%$ \\
\hline$T i$ & * & ICP.AES & $0.01 \%$ & $10 \%$ \\
\hline$\pi$ & 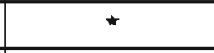 & ICP-AES & $10 \mathrm{ppm}$ & $1 \%$ \\
\hline$U$ & & ICP-AES & $10 \mathrm{ppm}$ & $1 \%$ \\
\hline $\mathrm{V}$ & & ICP-AES & $1 \mathrm{ppm}$ & $1 \%$ \\
\hline$w$ & * & ICP.AES & $10 \mathrm{ppm}$ & $1 \%$ \\
\hline $\mathrm{Zn}$ & & ICP-AES & $2 \mathrm{ppm}$ & $1 \%$ \\
\hline
\end{tabular}

